\title{
Two-frequency radiative transfer: Maxwell equations in random dielectrics
}

\author{
Albert C. Fannjiang* \\ Department of Mathematics, University of California, Davis, Davis, California 95616-8633 \\ *fannjiang@math.ucdavis.edu
}

Received July 6, 2007; accepted September 11, 2007;

posted October 1, 2007 (Doc. ID 84859); published November 8, 2007

\begin{abstract}
The paper addresses the space-frequency correlations of electromagnetic waves in general random, bianisotropic media whose constitutive tensors are complex Hermitian matrices. The two-frequency Wigner distribution (2f-WD) for polarized waves is introduced to describe the space-frequency correlations, and the closed form Wigner-Moyal equation is derived from the Maxwell equations. Two-frequency radiative transfer (2f-RT) equations are then derived from the Wigner-Moyal equation by using the multiple-scale expansion. For the simplest isotropic medium, the result coincides with Chandrasekhar's transfer equation. In birefringent media, the 2f-RT equations take the scalar form due to the absence of depolarization. A number of birefringent media such as chiral, uniaxial, and gyrotropic media are examined. For the unpolarized wave in an isotropic medium the $2 \mathrm{f}-\mathrm{RT}$ equations reduces to the $2 \mathrm{f}-\mathrm{RT}$ equation previously derived in part I of this research [J. Opt. Soc. Am. A 24, 2248 (2007)]. A similar Fokker-Planck-type equation is derived from the scalar 2f-RT equation for the birefringent media. (C) 2007 Optical Society of America
\end{abstract}

OCIS codes: $030.5620,290.4210$.

\section{INTRODUCTION}

In part I [1] of the series we studied the space-frequency correlation for scalar waves in random media as governed by the Helmholtz equation with a randomly fluctuating refractive index. To this end, we introduced the twofrequency Wigner distribution (2f-WD), which in the unscaled form is

$$
\begin{aligned}
W\left(\mathbf{x}, \mathbf{p} ; \omega_{1}, \omega_{2}\right)= & \frac{1}{(2 \pi)^{3}} \int e^{-i \mathbf{p}^{\dagger} \mathbf{y}} U_{1}\left(\frac{\mathbf{x}}{\omega_{1}}+\frac{\mathbf{y}}{2 \omega_{1}}\right) \\
& \times U_{2}^{*}\left(\frac{\mathbf{x}}{\omega_{2}}-\frac{\mathbf{y}}{2 \omega_{2}}\right) \mathrm{d} \mathbf{y},
\end{aligned}
$$

where $U_{1}$ and $U_{2}$ are the wave fields at frequencies $\omega_{1}$ and $\omega_{2}$, respectively. Throughout, ${ }^{\dagger}$ denotes the Hermitian conjugation of vectors or matrices and * denotes the complex conjugation. The important characteristic of definition (1) is that the spatial argument of each wave field is scaled in proportion to the respective wavelength. The variables $\mathbf{x}$ are the so-called size parameter in scattering theory when the phase velocity is unity [2].

In the weak-coupling (disorder) regime we derived the two-frequency radiative transfer (2f-RT) equation for the two-frequency Wigner distribution. We considered several approximations, notably the geometrical optics and paraxial approximations. Based on the dimensional analysis of these asymptotic equations, we obtained scaling behavior of the coherence bandwidth and coherence length. We also obtained the space-frequency correlation below the transport mean free path by analytically solving one of the paraxial $2 \mathrm{f}-\mathrm{RT}$ equations.

The main advantage of the $2 \mathrm{f}-\mathrm{RT}$ theory over the traditional equal-time RT theory is that it describes not just the energy transport but also the two space-time point mutual coherence in the following way. Let $u\left(t_{j}, \mathbf{x}_{j}\right), j$ $=1,2$ be the time-dependent wave field at two space-time points $\left(t_{j}, \mathbf{x}_{j}\right), j=1,2$. Let $\mathbf{x}=\left(\omega_{1} \mathbf{x}_{1}+\omega_{2} \mathbf{x}_{2}\right) / 2$ and $\mathbf{y}=\omega_{1} \mathbf{x}_{1}$ $-\omega_{2} \mathbf{x}_{2}$. Then we have

$$
\begin{aligned}
& \left\langle u\left(t_{1}, \mathbf{x}_{1}\right) u^{*}\left(t_{2}, \mathbf{x}_{2}\right)\right\rangle \\
& =\int e^{i\left(\omega_{2} t_{2}-\omega_{1} t_{1}\right)}\left\langle U_{1}\left(\mathbf{x}_{1}\right) U_{2}^{*}\left(\mathbf{x}_{2}\right)\right\rangle \mathrm{d} \omega_{1} \mathrm{~d} \omega_{2} \\
& =\int e^{i \mathbf{p}^{\dagger} \mathbf{y}} e^{-i \omega^{\prime} t} e^{-i \tau \omega}\left\langle W\left(\mathbf{x}, \mathbf{p} ; \omega+\omega^{\prime} / 2, \omega-\omega^{\prime} / 2\right)\right\rangle \\
& \quad \times \mathrm{d} \omega d \omega^{\prime} \mathrm{d} \mathbf{p},
\end{aligned}
$$

with $\quad t=\left(t_{1}+t_{2}\right) / 2, \tau=t_{1}-t_{2}, \omega=\left(\omega_{1}+\omega_{2}\right) / 2, \omega^{\prime}=\omega_{1}-\omega_{2}$. Here and below $\langle\cdot\rangle$ is the ensemble averaging w.r.t. the medium fluctuations. In comparison, the single-time correlation gives rise to the expression

$$
\begin{aligned}
& \left\langle u\left(t, \mathbf{x}_{1}\right) u^{\dagger}\left(t, \mathbf{x}_{2}\right)\right\rangle \\
& =\int e^{i \mathbf{p}^{\dagger} \mathbf{y}} e^{-i \omega^{\prime} t}\left[\int\left\langle W\left(\mathbf{x}, \mathbf{p} ; \omega+\omega^{\prime} / 2, \omega-\omega^{\prime} / 2\right)\right\rangle \mathrm{d} \omega\right] \\
& \quad \times \mathrm{d} \omega^{\prime} d \mathbf{p},
\end{aligned}
$$

which, through spectral decomposition, determines only the central-frequency-integrated 2f-WD. For a statistically stationary signal, Eq. (1) would be a function of $\tau$ only. In this case different frequency components are uncorrelated and consequently only the equal-frequency WD is necessary to describe the two-space-time correlation [3]. For statistically nonstationary signals the twofrequency cross correlation is needed to characterize the two-space-time correlation. 
The 2f-RT theory developed in part I has enabled precise estimation of important physical quantities such as the coherence length and the coherence bandwidth [1], which are medium characteristics relevant to communications and imaging in disordered media [4,5]. In particular, the two-frequency formulation is an indispensable tool for the statistical stability analysis of the time-reversal communication scheme with broadband signals in multiplescattering media (see [4], where a $2 \mathrm{f}-\mathrm{RT}$ equation and its solution play a key role). The $2 \mathrm{f}$-RT theory developed here is expected to extend these results to the case of polarized waves.

The organization of this paper is as follows. In Section 2 and Appendix A, we develop the two-frequency formulation of the Maxwell equations for a general heterogeneous dielectric in terms of $2 \mathrm{f}-\mathrm{WD}$. In Section 3 , we formulate the weak-coupling scaling limit for two-frequency Wigner-Moyal equation. In Section 4 we develop the multiscale expansion to find an approximate solution in the weak-coupling regime. In Section 5 and Appendix B, based on a solvability condition we give an explicit form to the 2f-RT equations for general bianisotropic media and in Subsection 5.A we derive a scalar 2f-RT equation for birefringent media. In Subsection 6.A, we consider the isotropic medium and show that the general $2 \mathrm{f}-\mathrm{RT}$ equations after a change of variable reduces to the two-frequency version of Chandrasekhar's transfer equation. In Subsections 6.B-6.D, we examine three birefringent media: chiral, uniaxial, and gyrotropic media. In Section 7, we analyze the unpolarized wave in the isotropic medium in the geometrical optics regime and show that the twofrequency version of Chandrasekhar's equation reduces to a Fokker-Planck-type equation rigorously derivable from the geometrical optics of the scalar wave [6]. We derive a similar equation from the scalar $2 \mathrm{f}-\mathrm{RT}$ equation for the birefringent media. We conclude the paper in Section 8 with a brief discussion on expressing the two-space-time correlation in terms of solutions of the $2 \mathrm{f}-\mathrm{RT}$ equations.

\section{MAXWELL EQUATIONS AND WIGNER-MOYAL EQUATIONS}

In this paper, we consider the electromagnetic wave propagation in a heterogeneous, lossless, bianisotropic dielectric medium. We assume that the scattering medium is free of charges and currents and start with the sourcefree Maxwell equations in the frequency domain,

$$
-i \omega \mathbf{K}\left[\begin{array}{l}
\mathbf{E} \\
\mathbf{H}
\end{array}\right]+\left[\begin{array}{cc}
0 & -\nabla \times \\
\nabla \times & 0
\end{array}\right]\left[\begin{array}{l}
\mathbf{E} \\
\mathbf{H}
\end{array}\right]=0,
$$

where $\mathbf{K}$ is, by the assumption of losslessness, a Hermitian matrix [7],

$$
\mathbf{K}=\left[\begin{array}{cc}
\mathbf{K}^{\epsilon} & \mathbf{K}^{\chi} \\
\mathbf{K}^{\chi^{\dagger}} & \mathbf{K}^{\mu}
\end{array}\right],
$$

with the permittivity and permeability tensors $\mathbf{K}^{\epsilon}, \mathbf{K}^{\mu}$, and the magnetoelectric tensor $\mathbf{K}^{\chi}$ [8]. The Hermitian matrix $\mathbf{K}$ is assumed to be always invertible. Here and below, $\nabla \times$ denotes the curl operator.

In an isotropic dielectric, $\mathbf{K}^{\epsilon}=\epsilon \mathbf{I}, \mathbf{K}^{\mu}=\mu \mathbf{I}, \mathbf{K}^{\chi}=0$. In a biisotropic dielectric, $\mathbf{K}^{\chi}$ as well as $\mathbf{K}^{\epsilon}, \mathbf{K}^{\mu}$ are nonzero sca- lars. A reciprocal chiral medium is biisotropic with purely imaginary $\mathbf{K}^{\chi}=i \chi$. The appearance of nonzero $\mathbf{K}^{\chi}$ arises from the so called magnetoelectric effect [9]. Crystals are often naturally anisotropic, and in some media (such as liquid crystals) it is possible to induce anisotropy by applying, e.g., an external electric field. In crystal optics, $\mathbf{K}^{\epsilon}, \mathbf{K}^{\mu}$ are real, symmetric matrices and $\mathbf{K}^{\chi}=0$ [10]. In response to a magnetic field, some materials can have a dielectric tensor that is complex-Hermitian; this is called the gyrotropic effect. In general, a magnetoelectric, bianisotropic medium has a constitutive tensor (3) with complex Hermitian $\mathbf{K}^{\epsilon}, \mathbf{K}^{\mu}$ and a complex matrix $\mathbf{K}^{\chi}$ satisfying the Post constraint [11]. It has been shown that a moving medium, even isotropic, must be treated as bianisotropic [9,12].

In general, $\mathbf{K}$ is a function of the frequency $\omega$ (for dispersive media), but it turns out that if the frequencydependence of $\mathbf{K}$ is sufficiently smooth, the $2 \mathrm{f}-\mathrm{RT}$ equations derived in the present framework have the same form as for nondispersive media; the frequency dependence would enter the coefficients of the equations in the obvious way [1]. For simplicity of presentation we shall assume that the medium is nondispersive.

Writing the total field $\mathbf{U}=(\mathbf{E}, \mathbf{H})$, we introduce the twofrequency matrix-valued Wigner distribution,

$$
\begin{aligned}
& \mathbf{W}\left(\mathbf{x}, \mathbf{p} ; \omega_{1}, \omega_{2}\right) \\
& \quad=\frac{1}{(2 \pi)^{3}} \int e^{-i \mathbf{p}^{\dagger} \mathbf{y}} \mathbf{U}_{1}\left(\frac{\mathbf{x}}{\omega_{1}}+\frac{\mathbf{y}}{2 \omega_{1}}\right) \mathbf{U}_{2}^{\dagger}\left(\frac{\mathbf{x}}{\omega_{2}}-\frac{\mathbf{y}}{2 \omega_{2}}\right) \mathrm{d} \mathbf{y},
\end{aligned}
$$

where $\mathbf{U}_{1}$ and $\mathbf{U}_{2}$ are the total fields at frequencies $\omega_{1}$ and $\omega_{2}$, respectively. From the definition we see that the variables $\mathbf{x}$ and $\mathbf{p}^{-1}$ have the dimension of length/time. Although the scaling factors in the arguments of $\mathbf{U}_{1}$ and $\mathbf{U}_{2}$ are not required for the development of the $2 \mathrm{f}-\mathrm{RT}$ theory for the first-order (Maxwell) equations, they are particularly useful in the case of the second-order (Helmholtz and paraxial wave) equations. For consistency and continuity of presentation (see Section 7) we work with definition (4) in the present paper. For an alternative development of the 2f-RT theory for Maxwell's equations in terms of the 2f-WD without the scaling factors, we refer the reader to [13].

First note the symmetry of the Wigner distribution matrix:

$$
\mathbf{W}^{\dagger}\left(\mathbf{x}, \mathbf{p} ; \omega_{1}, \omega_{2}\right)=\mathbf{W}\left(\mathbf{x}, \mathbf{p} ; \omega_{2}, \omega_{1}\right) .
$$

In other words, the right-hand side of Eq. (4) is invariant under the simultaneous transformations of Hermitian conjugation ${ }^{\dagger}$ and frequency exchange $\omega_{1} \leftrightarrow \omega_{2}$.

In what follows we shall omit writing the arguments of any fields if there is no risk of confusion.

We put Eq. (5) in the form of a general symmetric hyperbolic system [14,15],

$$
-i \omega \mathbf{K U}+\mathbf{R}_{l} \partial_{x_{l}} \mathbf{U}=0
$$

where the symmetric matrices $\mathbf{R}_{j}$ are given by

$$
\mathbf{R}_{j}=\left[\begin{array}{cc}
0 & \mathbf{T}_{j} \\
-\mathbf{T}_{j} & 0
\end{array}\right],
$$

with 


$$
\mathbf{T}_{1}=\left[\begin{array}{ccc}
0 & 0 & 0 \\
0 & 0 & -1 \\
0 & 1 & 0
\end{array}\right], \quad \mathbf{T}_{2}=\left[\begin{array}{ccc}
0 & 0 & 1 \\
0 & 0 & 0 \\
-1 & 0 & 0
\end{array}\right], \quad \mathbf{T}_{3}=\left[\begin{array}{ccc}
0 & -1 & 0 \\
1 & 0 & 0 \\
0 & 0 & 0
\end{array}\right] .
$$

The matrices $i T_{j}, j=1,2,3$ are related to the photon spin matrices [16].

Throughout this paper the dot notation, ".", is used exclusively for the directional derivative as in $\mathbf{p} \cdot \nabla=p_{j} \partial_{x_{j}}$. All vectors are treated as matrices, and the scalar product is just the matrix multiplication between row and column vectors. All vectors are taken to be, by default, column vectors, unless explicitly transposed. Einstein's summation is applied to all english indices.

Applying the operator $\mathbf{R}_{j} \partial / \partial x_{j}$ to $\mathbf{W}$ and using Eq. (6) we obtain

$$
\mathbf{R}_{j} \frac{\partial}{\partial x_{j}} \mathbf{W}=-2 i p_{j} \mathbf{R}_{j} \mathbf{W}+2 i \int e^{i \mathbf{q}^{\dagger} \mathbf{x} / \omega_{1}} \hat{\mathbf{K}}(\mathbf{q}) \mathbf{W}\left(\mathbf{x}, \mathbf{p}-\frac{\mathbf{q}}{2 \omega_{1}}\right) \mathrm{d} \mathbf{q},
$$

whose derivation is given in Appendix A. From Eqs. (7) and (5) we also have

$$
\frac{\partial}{\partial x_{j}} \mathbf{W} \mathbf{R}_{j}^{\dagger}=2 i \mathbf{W} p_{j} \mathbf{R}_{j}-2 i \int \mathbf{W}\left(\mathbf{x}, \mathbf{p}+\frac{\mathbf{q}}{2 \omega_{2}}\right) \hat{\mathbf{K}}(\mathbf{q}) e^{i \mathbf{q}^{\dagger} \mathbf{x} / \omega_{2}} \mathrm{~d} \mathbf{q} .
$$

Here and below, $\hat{\mathbf{K}}$ stands for the Fourier transform (spectral density) of $\mathbf{K}$ as in

$$
\mathbf{K}(\mathbf{x})=\int e^{i \mathbf{x}^{\dagger} \mathbf{q} \hat{\mathbf{K}}}(\mathbf{q}) \mathrm{d} \mathbf{q} .
$$

For a Hermitian $\mathbf{K}$ we have $\hat{\mathbf{K}}(\mathbf{p})=\hat{\mathbf{K}}^{\dagger}(-\mathbf{p}), \forall \mathbf{p}$.

\section{WEAK-COUPLING LIMIT}

As in part I [1] we consider the weak-coupling regime with the tensor

$$
\mathbf{K}(\mathbf{x})=\mathbf{K}_{0}(\mathbf{I}+\sqrt{\ell} \mathbf{V}(\mathbf{x} / \ell)), \quad \ell \ll 1,
$$

where the Hermitian matrix $\mathbf{K}_{0}$ represents the uniform background medium and $\sqrt{\ell} \mathbf{V}$ represents the relative fluctuations of the permittivity-permeability tensor. The small parameter $l$ describes the ratio of the scale of the medium fluctuation to the propagation distance. In an isotropic dielectric,

$$
\mathbf{K}_{0}=\left[\begin{array}{cc}
\epsilon_{0} \mathbf{I}_{3} & 0 \\
0 & \mu_{0} \mathbf{I}_{3}
\end{array}\right], \quad \mathbf{V}=\left[\begin{array}{cc}
\tilde{\epsilon}_{3} & 0 \\
0 & \tilde{\mu} \mathbf{I}_{3}
\end{array}\right],
$$

where $\tilde{\boldsymbol{\epsilon}}$ and $\tilde{\mu}$ are the electric and magnetic susceptibility, respectively. In general $\mathbf{K}_{0}$ is a Hermitian matrix, and its blocks, as in Eq. (3), are denoted by $\mathbf{K}_{0}^{\epsilon}, \mathbf{K}_{0}^{\mu}, \mathbf{K}_{0}^{\chi}, \mathbf{K}_{0}^{\chi \dagger}$, respectively. To preserve the Hermiticity of $\mathbf{K}$ and $\mathbf{K}_{0}$ the matrix $\mathbf{V}$ must satisfy

$$
\mathbf{V}^{\dagger} \mathbf{K}_{0}=\mathbf{K}_{0} \mathbf{V} .
$$

We shall assume below that $\mathbf{K}_{0}$ is either positive or negative definite. Otherwise, the materials would be lossy since the refractive index is not real valued if $\mathbf{K}_{0}$ is not sign definite. A negative-definite $\mathbf{K}_{0}$ gives rise to negative refractive index, which is a hot topic in metamaterial research [17-19]. To fix the idea, let us take $\mathbf{K}_{0}$ to be positive definite. With minor notational change, our method applies equally well to the negative definite case.

We assume that $\mathbf{V}=\left[V_{i j}\right]$ is a statistically homogeneous random field with the spectral density tensors $\boldsymbol{\Phi}$ $=\left[\Phi_{i j m n}\right], \boldsymbol{\Psi}=\left[\Psi_{i j m n}\right]$ such that

$$
\begin{aligned}
& \left\langle V_{i j}(\mathbf{x}) V_{m n}^{*}(\mathbf{y})\right\rangle=\int e^{i \mathbf{k}^{\dagger}(\mathbf{x}-\mathbf{y})} \Phi_{i j m n}(\mathbf{k}) \mathrm{d} \mathbf{k} \\
& \left\langle V_{i j}(\mathbf{x}) V_{m n}(\mathbf{y})\right\rangle=\int e^{i \mathbf{k}^{\dagger}(\mathbf{x}-\mathbf{y})} \Psi_{i j m n}(\mathbf{k}) \mathrm{d} \mathbf{k} .
\end{aligned}
$$

This implies the following relations:

$$
\begin{aligned}
& \left\langle\hat{V}_{i j}(\mathbf{p}) \hat{V}_{m n}^{*}(\mathbf{q})\right\rangle=\Phi_{i j m n}(\mathbf{p}) \delta(\mathbf{p}-\mathbf{q}), \\
& \left\langle\hat{V}_{i j}(\mathbf{p}) \hat{V}_{m n}(\mathbf{q})\right\rangle=\Psi_{i j m n}(\mathbf{p}) \delta(\mathbf{p}+\mathbf{q}) .
\end{aligned}
$$

In the case of real-valued $\mathbf{V}, \boldsymbol{\Phi}=\boldsymbol{\Psi}$. The spectral density tensors have the basic symmetry

$$
\begin{gathered}
\Phi_{i j m n}^{*}(\mathbf{p})=\Phi_{m n i j}(\mathbf{p}), \\
\Psi_{i j m n}(-\mathbf{p})=\Psi_{m n i j}(\mathbf{p}),
\end{gathered}
$$

Furthermore, Eq. (10) implies that

$$
\begin{aligned}
& K_{0, i j} \Psi_{m n j l}(\mathbf{p})=K_{0, l j}^{*} \Phi_{m n j i}(\mathbf{p}), \\
& K_{0, i j} \Phi_{m n j l}(\mathbf{p})=K_{0, l j}^{*} \Psi_{m n j i}(\mathbf{p}) .
\end{aligned}
$$

As in part I, we consider the regime where the wavelengths are of the same order of magnitude as the correlation length of the medium fluctuations by rescaling the frequencies $\omega_{j}=\widetilde{\omega}_{j} / \ell, j=1,2$. This choice of frequency scaling results in strong scattering by the medium heterogeneities. For ease of notation, we drop the tilde in $\widetilde{\omega}_{j}$ below. To capture the high-frequency behavior of the wave field, we redefine the $2 \mathrm{f}-\mathrm{WD}$ as

$$
\mathbf{W}(\mathbf{x}, \mathbf{p})=\frac{1}{(2 \pi)^{3}} \int e^{-i \mathbf{p}^{\dagger} \mathbf{y}} \mathbf{U}_{1}\left(\frac{\mathbf{x}}{\omega_{1}}+\frac{\ell \mathbf{y}}{2 \omega_{1}}\right) \mathbf{U}_{2}^{\dagger}\left(\frac{\mathbf{x}}{\omega_{2}}-\frac{\ell \mathbf{y}}{2 \omega_{2}}\right) \mathrm{d} \mathbf{y} .
$$

We also assume that $\omega_{1}, \omega_{2} \rightarrow \omega$ as $\ell \rightarrow 0$ such that

$$
\frac{\omega_{2}-\omega_{1}}{\omega \ell}=\beta
$$

with a fixed constant $\beta$. The governing equations for Eq. (19) become

$$
\begin{aligned}
\mathbf{R}_{j} \frac{\partial}{\partial x_{j}} \mathbf{W}= & -\frac{2 i}{\ell} p_{j} \mathbf{R}_{j} \mathbf{W}+\frac{2 i}{\ell} \mathbf{K}_{0} \mathbf{W} \\
& +\frac{2 i}{\sqrt{\ell}} \int e^{i \mathbf{q}^{\dagger} \tilde{\mathbf{x}} / \omega_{1}} \mathbf{K}_{0} \hat{\mathbf{V}}(\mathbf{q}) \mathbf{W}\left(\mathbf{p}-\frac{\mathbf{q}}{2 \omega_{1}}\right) \mathrm{d} \mathbf{q},
\end{aligned}
$$




$$
\begin{aligned}
\frac{\partial}{\partial x_{j}} \mathbf{W R}_{j}= & \frac{2 i}{\ell} \mathbf{W} p_{j} \mathbf{R}_{j}-\frac{2 i}{\ell} \mathbf{W} \mathbf{K}_{0} \\
& -\frac{2 i}{\sqrt{\ell}} \int \mathbf{W}\left(\mathbf{p}-\frac{\mathbf{q}}{2 \omega_{2}}\right) \hat{\mathbf{V}}^{\dagger}(\mathbf{q}) \mathbf{K}_{0} e^{-i \mathbf{q} \mathbf{q}^{\dagger} \tilde{\mathbf{x}} / \omega_{2}} \mathbf{d} \mathbf{q},
\end{aligned}
$$

where $\widetilde{\mathbf{x}}=\mathbf{x} / \ell$ is the fast spatial variable. In order to cancel the background effect, we multiply Eq. (21) by $K_{0}^{-1}$ from the left and Eq. (22) by $K_{0}^{-1}$ from the right and add them to obtain the symmetrical form

$$
\begin{aligned}
& \mathbf{K}_{0}^{-1} \mathbf{R}_{j} \frac{\partial}{\partial x_{j}} \mathbf{W}+\frac{\partial}{\partial x_{j}} \mathbf{W R}_{j} \mathbf{K}_{0}^{-1}+\frac{2 i}{\ell}\left[\mathbf{K}_{0}^{-1} p_{j} \mathbf{R}_{j} \mathbf{W}-\mathbf{W} p_{j} \mathbf{R}_{j} \mathbf{K}_{0}^{-1}\right] \\
& =\frac{2 i}{\sqrt{\ell}} \int\left[e^{i \mathbf{q}+\tilde{\mathbf{x}} / \omega_{1}} \hat{\mathbf{V}}(\mathbf{q}) \mathbf{W}\left(\mathbf{p}-\frac{\mathbf{q}}{2 \omega_{1}}\right)\right. \\
& \left.-\mathbf{W}\left(\mathbf{p}-\frac{\mathbf{q}}{2 \omega_{2}}\right) \hat{\mathbf{V}}^{\dagger}(\mathbf{q}) e^{-i \mathbf{q}^{\dagger} \tilde{\mathbf{x}} / \omega_{2}}\right] \mathrm{d} \mathbf{q} .
\end{aligned}
$$

This is the equation that we shall work with to derive the 2f-RT equations employing the multiscale expansion (MSE) $[1,15]$. Note that Eq. (23) is invariant under the simultaneous transformations of Hermitian conjugation ${ }^{\dagger}$ and frequency exchange $\omega_{1} \leftrightarrow \omega_{2}$.

If, instead of adding the two equations, we subtract them, then we obtain the antisymmetric form

$$
\begin{aligned}
-\frac{4 i}{\ell} \mathbf{W} & +\mathbf{K}_{0}^{-1} \mathbf{R}_{j} \frac{\partial}{\partial x_{j}} \mathbf{W}-\frac{\partial}{\partial x_{j}} \mathbf{W} \mathbf{R}_{j} \mathbf{K}_{0}^{-1}+\frac{2 i}{\ell}\left[\mathbf{K}_{0}^{-1} p_{j} \mathbf{R}_{j} \mathbf{W}\right. \\
+ & \left.\mathbf{W} p_{j} \mathbf{R}_{j} \mathbf{K}_{0}^{-1}\right] \\
= & \frac{2 i}{\sqrt{\ell}} \int\left[e^{i \mathbf{q}^{\dagger} \tilde{\mathbf{x}} / \omega_{1}} \hat{\mathbf{V}}(\mathbf{q}) \mathbf{W}\left(\mathbf{p}-\frac{\mathbf{q}}{2 \omega_{1}}\right)\right. \\
& \left.+\mathbf{W}\left(\mathbf{p}-\frac{\mathbf{q}}{2 \omega_{2}}\right) \hat{\mathbf{V}}^{\dagger}(\mathbf{q}) e^{-i \mathbf{q}^{\dagger} \mathbf{\mathbf { x }} / \omega_{2}}\right] \mathrm{d} \mathbf{q} .
\end{aligned}
$$

Equation (24) requires a different treatment and will not be pursued here. However, the leading order $\ell^{-1}$ terms of Eq. (24) impose a constraint, which will be discussed in the Conclusion.

\section{MULTISCALE EXPANSION}

The key point of MSE is to separate the fast variable $\widetilde{\mathbf{x}}$ from the slow variable $\mathbf{x}$ and make the substitution

$$
\begin{aligned}
& \mathbf{R}_{j} \partial_{x_{j}} \mathbf{W} \rightarrow \mathbf{R}_{j} \frac{\partial}{\partial x_{j}} \mathbf{W}+\ell^{-1} \mathbf{R}_{j} \frac{\partial}{\partial \widetilde{x}_{j}} \mathbf{W}, \\
& \partial_{x_{j}} \mathbf{W} \mathbf{R}_{j} \rightarrow \frac{\partial}{\partial x_{j}} \mathbf{W} \mathbf{R}_{j}+\ell^{-1} \frac{\partial}{\partial \widetilde{x}_{j}} \mathbf{W} \mathbf{R}_{j} .
\end{aligned}
$$

The idea is that for sufficiently small $\ell$ the two widely separated scales, represented by $\mathbf{x}$ and $\widetilde{\mathbf{x}}$ respectively, become mathematically (but not physically) independent.
We posit the expansion $\mathbf{W}=\overline{\mathbf{W}}+\sqrt{\ell} \mathbf{W}_{1}+\ell \mathbf{W}_{2}+\cdots$, substitute it into Eq. (23), and equate terms of same order of magnitude.

\section{A. Leading Term}

The $\ell^{-1}$ terms yield

$$
\mathbf{K}_{0}^{-1} \mathbf{R}_{j} \frac{\partial}{\partial \widetilde{x}_{j}} \overline{\mathbf{W}}+\frac{\partial}{\partial \widetilde{x}_{j}} \overline{\mathbf{W}} \mathbf{R}_{j} \mathbf{K}_{0}^{-1}+2 i\left[\mathbf{K}_{0}^{-1} p_{j} \mathbf{R}_{j} \overline{\mathbf{W}}-\overline{\mathbf{W}} p_{j} \mathbf{R}_{j} \mathbf{K}_{0}^{-1}\right]=0 .
$$

We hypothesize that the leading order term $\overline{\mathbf{W}}$ $=\overline{\mathbf{W}}(\mathbf{x}, \mathbf{p})$ is independent of the fast variable $\widetilde{\mathbf{x}}$. Thus the first two terms of Eq. (25) vanish so the equation reduces to

$$
\mathbf{K}_{0}^{-1} p_{j} \mathbf{R}_{j} \overline{\mathbf{W}}-\overline{\mathbf{W}} p_{j} \mathbf{R}_{j} \mathbf{K}_{0}^{-1}=0 .
$$

Equation (26) arises also in the equal-time RT theory [15] and can be solved as follows. For a positive (or negative) definite $\mathbf{K}_{0}$, consider the eigenvalues $\left\{\Omega^{\sigma}\right\}$ and eigenvectors $\left\{\mathbf{e}^{\sigma, \alpha}\right\}$ of the matrix $\mathbf{K}_{0}^{-1} p_{j} \mathbf{R}_{j}$, where the index $\alpha$ keeps track of the multiplicity and hence depends on $\sigma$. As $\mathbf{K}_{0}^{-1} p_{j} \mathbf{R}_{j}$ is Hermitian with respect to the scalar product defined by $\mathbf{a}^{\dagger} \mathbf{K}_{0} \mathbf{b}, \forall \mathbf{a}, \mathbf{b} \in \mathrm{C}^{6}$, the eigenvalues are real and the eigenvectors form a complete set of $\mathbf{K}_{0}$-orthogonal basis in $\mathbb{C}^{6}$. Alternatively, we may work with the Hermitian matrix $\mathbf{K}_{0}^{-1 / 2} p_{j} \mathbf{R}_{j} \mathbf{K}_{0}^{-1 / 2}$ in the image space, with the standard scalar product, under the transformation $\mathbf{K}_{0}^{1 / 2}$. Let the eigenvectors $\left\{\mathbf{e}^{\sigma, \alpha}\right\}$ be normalized such that $\mathbf{e}^{\sigma, \alpha \dagger} \mathbf{K}_{0} \mathbf{e}^{\tau, \zeta}=\delta_{\sigma, \tau} \delta_{\alpha, \zeta}$.

Clearly, the eigenvalues $\Omega^{\sigma}$ as a function of the wave vector $\mathbf{p}$ define the dispersion relations. For general bianisotropic dielectric, it is easy to check that $\Omega^{0}=0$ is always an eigenvalue with eigenvectors

$$
\mathbf{e}^{0,1}(\mathbf{p}) \sim\left(\begin{array}{l}
\mathbf{p} \\
0
\end{array}\right), \quad \mathbf{e}^{0,2}(\mathbf{p}) \sim\left(\begin{array}{l}
0 \\
\mathbf{p}
\end{array}\right) .
$$

Since $\mathbf{K}_{0}$ is invertible, it follows that the null space of $\mathbf{K}_{0}^{-1} p_{j} \mathbf{R}_{j}$ is spanned by these two nonpropagating modes. It is easy to check that $\left\{\mathbf{d}^{\sigma, \alpha \dagger}(\mathbf{p}): \mathbf{d}^{\sigma, \alpha}(\mathbf{p})=\mathbf{K}_{0} \mathbf{e}^{\sigma, \alpha}(\mathbf{p})\right\}$ are

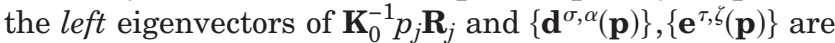
co-orthogonal with respect to the standard scalar product:

$$
\mathbf{d}^{\sigma, \alpha \dagger}(\mathbf{p}) \mathbf{e}^{\tau, \zeta}(\mathbf{p})=\delta_{\sigma, \tau} \delta_{\alpha, \zeta} .
$$

This relation will be useful in deriving the $2 \mathrm{f}-\mathrm{RT}$ equations (see Appendix B).

Throughout, the english indices represent the spatial degrees of freedom while the greek indices represent the modal and polarization degrees of freedom. It is important to keep this distinction in mind in the subsequent analysis. The Einstein summation convention and the Hermitian conjugation are used only on the arabic indices.

It can be checked easily that the general solution to Eq. (26) is given by [15]

$$
\overline{\mathbf{W}}(\mathbf{x}, \mathbf{p})=\sum_{\sigma, \alpha, \zeta} \bar{W}_{\alpha \zeta}^{\sigma}(\mathbf{x}, \mathbf{p}) \mathbf{E}^{\sigma, \alpha \zeta}(\mathbf{p}, \mathbf{p})
$$

where $\bar{W}_{\alpha \zeta}^{\sigma}$ are generally complex-valued functions and 


$$
\mathbf{E}^{\sigma, \alpha \zeta}(\mathbf{p}, \mathbf{q})=\mathbf{e}^{\sigma, \alpha}(\mathbf{p}) \mathbf{e}^{\sigma, \zeta^{\dagger}}(\mathbf{q}) .
$$

Likewise, we define

$$
\mathbf{D}^{\sigma, \alpha \zeta}(\mathbf{p}, \mathbf{q})=\mathbf{d}^{\sigma, \alpha}(\mathbf{p}) \mathbf{d}^{\sigma, \zeta^{\dagger}}(\mathbf{q}) .
$$

The linear span of $\left\{\mathbf{E}^{\tau, \alpha \zeta}(\mathbf{p}, \mathbf{p}), \forall \tau, \alpha, \zeta, \mathbf{p}\right\}$ is a Hilbert space, denoted by $\mathfrak{M}_{\mathbf{p}}$, for each $\mathbf{p} \neq 0$ with the scalar product $\operatorname{Tr}\left[\mathbf{H}^{\dagger} \mathbf{K G K}\right], \mathbf{H}, \mathbf{G} \in \mathfrak{M}_{\mathbf{p}}$. The matrices $\overline{\mathbf{W}}^{\sigma}=\left[\bar{W}_{\alpha \zeta}^{\sigma}\right]$, free of the arabic indices, are called the coherence matrices.

For $\widetilde{\mathbf{x}}$-independent $\overline{\mathbf{W}}$, the constraint that the electric displacement and the magnetic induction both be divergence free yields, on the macroscopic scale,

$$
( \pm \nabla, \pm \nabla) \cdot \mathbf{K}_{0} \overline{\mathbf{W}}=0
$$

which, in view of definition (19), is equivalent to

$$
\left( \pm \mathbf{p}^{\dagger}, \pm \mathbf{p}^{\dagger}\right) \mathbf{K}_{0} \overline{\mathbf{W}}(\mathbf{x}, \mathbf{p})=0
$$

Hence by Eq. (27), $\mathbf{d}^{0, j \dagger} \overline{\mathbf{W}}=0$ and by Eq. (28) $\overline{\mathbf{W}}^{0}=0$, where $\mathbf{W}^{0}$ in Eq. (29) is the coherence matrix associated with the nonpropagating mode $\Omega^{0}=0$.

\section{B. Correctors}

The $\ell^{-1 / 2}$ terms yield the equation

$$
\begin{aligned}
2 l \mathbf{W}_{1} & +\mathbf{K}_{0}^{-1} \mathbf{R}_{j} \frac{\partial}{\partial \widetilde{x}_{j}} \mathbf{W}_{1}+\frac{\partial}{\partial \widetilde{x}_{j}} \mathbf{W}_{1} \mathbf{R}_{j} \mathbf{K}_{0}^{-1} \\
+ & 2 i\left[\mathbf{K}_{0}^{-1} p_{j} \mathbf{R}_{j} \mathbf{W}_{1}-\mathbf{W}_{1} p_{j} \mathbf{R}_{j} \mathbf{K}_{0}^{-1}\right] \\
= & 2 i \int d \mathbf{q}\left[e^{i \mathbf{q}^{\dagger} \tilde{\mathbf{x}} / \omega_{1}} \hat{\mathbf{V}}(\mathbf{q}) \overline{\mathbf{W}}\left(\mathbf{p}-\frac{\mathbf{q}}{2 \omega_{1}}\right)\right. \\
& \left.-\overline{\mathbf{W}}\left(\mathbf{p}-\frac{\mathbf{q}}{2 \omega_{2}}\right) \hat{\mathbf{V}}^{\dagger}(\mathbf{q}) e^{-i \mathbf{q}^{\dagger} \tilde{\mathbf{x}} / \omega_{2}}\right]
\end{aligned}
$$

where, as in part I [1], we have added a small regularization term. The reader is referred to part I [1] for the discussion of the choice of the regularization parameter. Physically, the sign of the parameter (positive here) amounts to choosing the direction of causality.

We Fourier transform Eq. (33) in $\widetilde{\mathbf{x}}$,

$$
\begin{aligned}
& -i 2 \ell \hat{\mathbf{W}}_{1}(\mathbf{k}, \mathbf{p})+\mathbf{K}_{0}^{-1} k_{j} \mathbf{R}_{j} \hat{\mathbf{W}}_{1}(\mathbf{k}, \mathbf{p})+\hat{\mathbf{W}}_{1}(\mathbf{k}, \mathbf{p}) k_{j} \mathbf{R}_{j} \mathbf{K}_{0}^{-1} \\
& +2\left[\mathbf{K}_{0}^{-1} p_{j} \mathbf{R}_{j} \hat{\mathbf{W}}_{1}(\mathbf{k}, \mathbf{p})-\hat{\mathbf{W}}_{1}(\mathbf{k}, \mathbf{p}) p_{j} \mathbf{R}_{j} \mathbf{K}_{0}^{-1}\right] \\
& =2\left[\hat{\mathbf{V}}\left(\omega_{1} \mathbf{k}\right) \overline{\mathbf{W}}\left(\mathbf{p}-\frac{\mathbf{k}}{2}\right)-\overline{\mathbf{W}}\left(\mathbf{p}+\frac{\mathbf{k}}{2}\right) \hat{\mathbf{V}}^{\dagger}\left(-\omega_{2} \mathbf{k}\right)\right],
\end{aligned}
$$

and posit the solution

$$
\hat{\mathbf{W}}_{1}(\mathbf{k}, \mathbf{p})=\sum_{\sigma, \alpha, \zeta} C_{\alpha \zeta}^{\sigma}(\mathbf{k}, \mathbf{p}) \mathbf{E}^{\sigma, \alpha \zeta}\left(\mathbf{p}+\frac{\mathbf{k}}{2}, \mathbf{p}-\frac{\mathbf{k}}{2}\right),
$$

where $C_{\alpha \zeta}^{\sigma}$ are generally complex numbers. Note that the two arguments of $\mathbf{E}^{\sigma, \alpha \zeta}$ in Eq. (35) are at different momenta $\mathbf{p}+\mathbf{k} / 2, \mathbf{p}-\mathbf{k} / 2$.

We substitute Eqs. (29) and (35) into Eq. (34) and multiply by $\mathbf{d}^{\sigma, \alpha \dagger}(\mathbf{p}+\mathbf{k} / 2)$ from the left and with $\mathbf{d}^{\sigma, \zeta}(\mathbf{p}$ -k/2) from the right and solve the resulting equation algebraically. This yields the coefficients

$$
\begin{aligned}
C_{\alpha \zeta}^{\sigma}(\mathbf{k}, \mathbf{p})= & \left(\Omega^{\sigma}\left(\mathbf{p}+\frac{\mathbf{k}}{2}\right)-\Omega^{\sigma}\left(\mathbf{p}-\frac{\mathbf{k}}{2}\right)-i \ell\right)^{-1} \\
& \times \sum_{\eta}\left[\mathbf{d}^{\sigma, \alpha^{\dagger}}\left(\mathbf{p}+\frac{\mathbf{k}}{2}\right) \hat{\mathbf{V}}\left(\omega_{1} \mathbf{k}\right) \bar{W}_{\eta \zeta}^{\sigma}\left(\mathbf{p}-\frac{\mathbf{k}}{2}\right)\right. \\
& \times \mathbf{e}^{\sigma, \eta}\left(\mathbf{p}-\frac{\mathbf{k}}{2}\right)-\bar{W}_{\alpha \eta}^{\sigma}\left(\mathbf{p}+\frac{\mathbf{k}}{2}\right) \mathbf{e}^{\sigma, \eta^{\dagger}}\left(\mathbf{p}+\frac{\mathbf{k}}{2}\right) \\
& \left.\times \hat{\mathbf{V}}^{\dagger}\left(-\omega_{2} \mathbf{k}\right) \mathbf{d}^{\sigma, \zeta}\left(\mathbf{p}-\frac{\mathbf{k}}{2}\right)\right] .
\end{aligned}
$$

When the leading term $\overline{\mathbf{W}}$ is invariant under the simultaneous transformations of Hermitian conjugation $\dagger$ and frequency exchange $\omega_{1} \leftrightarrow \omega_{2}$, so is $\mathbf{W}_{1}$. This invariance is manifest in the relation

$$
C_{\zeta \alpha}^{\sigma^{*}}\left(-\mathbf{k}, \mathbf{p} ; \omega_{1}, \omega_{2}\right)=C_{\alpha \zeta}^{\sigma}\left(\mathbf{k}, \mathbf{p} ; \omega_{2}, \omega_{1}\right)
$$

Finally, the $O(1)$ terms yield the equation after adding a regularizing term $2 \ell \mathbf{W}_{2}$ :

$$
\begin{aligned}
2 \ell \mathbf{W}_{2} & +\mathbf{K}_{0}^{-1} \mathbf{R}_{j} \frac{\partial}{\partial \widetilde{x}_{j}} \mathbf{W}_{2}+\frac{\partial}{\partial \widetilde{x}_{j}} \mathbf{W}_{2} \mathbf{R}_{j} \mathbf{K}_{0}^{-1} \\
& +2 i\left[\mathbf{K}_{0}^{-1} p_{j} \mathbf{R}_{j} \mathbf{W}_{2}-\mathbf{W}_{2} p_{j} \mathbf{R}_{j} \mathbf{K}_{0}^{-1}\right]=\mathbf{F},
\end{aligned}
$$

with

$$
\begin{aligned}
\mathbf{F}= & 2 i \int \mathrm{d} \mathbf{q}\left[e^{i \mathbf{q}^{\dagger} \tilde{\mathbf{x}} / \omega_{1}} \hat{\mathbf{V}}(\mathbf{q}) \mathbf{W}_{1}\left(\mathbf{p}-\frac{\mathbf{q}}{2 \omega_{1}}\right)\right. \\
& \left.-\mathbf{W}_{1}\left(\mathbf{p}-\frac{\mathbf{q}}{2 \omega_{2}}\right) \hat{\mathbf{V}}^{\dagger}(\mathbf{q}) e^{-i \mathbf{q}^{\dagger} \tilde{\mathbf{x}} / \omega_{2}}\right] \\
& -\mathbf{K}_{0}^{-1} \mathbf{R}_{j} \frac{\partial}{\partial x_{j}} \overline{\mathbf{W}}-\frac{\partial}{\partial x_{j}} \overline{\mathbf{W}} \mathbf{R}_{j} \mathbf{K}_{0}^{-1} .
\end{aligned}
$$

Note again that $\mathbf{F}$ is invariant under the simultaneous transformations of Hermitian conjugation $\dagger$ and frequency exchange $\omega_{1} \leftrightarrow \omega_{2}$. We can, but need not, solve Eq. (37) explicitly as Eq. (34). However, in order for the second perturbation $\ell \mathbf{W}_{2}$ to vanish in the limit $\ell \rightarrow 0, \mathbf{F}$ must satisfy the solvability condition

$$
\lim _{\ell \rightarrow 0} \operatorname{Tr}\left\langle\mathbf{G}^{\dagger} \mathbf{K}_{0} \mathbf{F} \mathbf{K}_{0}\right\rangle=0
$$

for all random stationary matrices $\mathbf{G}$ satisfying Eq. (25). This can be seen by transforming Eq. (37) into $\operatorname{Tr}\left\langle\mathbf{G}^{\dagger} \mathbf{K}_{0}(37) \mathbf{K}_{0}\right\rangle$, which by Eq. (25) implies $2 \ell \operatorname{Tr}\left\langle\mathbf{G}^{\dagger} \mathbf{K}_{0} \mathbf{W}_{2} \mathbf{K}_{0}\right\rangle=\operatorname{Tr}\left\langle\mathbf{G}^{\dagger} \mathbf{K}_{0} \mathbf{F} \mathbf{K}_{0}\right\rangle$ and hence Eq. (39).

Fortunately, we do not need to work with the full solvability condition (39). It suffices to require that Eq. (39) to be fulfilled by all deterministic $\mathbf{G}$, independent of $\widetilde{\mathbf{x}}$, such that

$$
\mathbf{K}_{0}^{-1} p_{j} \mathbf{R}_{j} \mathbf{G}-\mathbf{G} p_{j} \mathbf{R}_{j} \mathbf{K}_{0}^{-1}=0 .
$$

In other words, as in Eq. (29), we consider only a subspace of the solution space of Eq. (25) and replace Eq. (39) with 


$$
\lim _{\ell \rightarrow 0} \operatorname{Tr}\left(\mathbf{D}^{\tau, \xi \nu^{\dagger}}(\mathbf{p}, \mathbf{p})\langle\mathbf{F}(\mathbf{x}, \widetilde{\mathbf{x}}, \mathbf{p})\rangle\right)=0, \quad \forall \tau, \xi, \nu, \mathbf{p}, \mathbf{x}, \widetilde{\mathbf{x}},
$$

where $\mathbf{D}^{\tau, \xi \nu}$ are defined in Eq. (31). As noted above, Eqs. (23), (33), and (38) are invariant under the simultaneous transformations of Hermitian conjugation $\dagger$ and frequency exchange $\omega_{1} \leftrightarrow \omega_{2}$, and therefore Eq. (41) must also be invariant under the same transformations.

To summarize, we have constructed the three-term expansion $\overline{\mathbf{W}}+\sqrt{\ell} \mathbf{W}_{1}+\ell \mathbf{W}_{2}$, which is an approximate solution of the $2 \mathrm{f}$ Wigner-Moyal equation in the sense that the left-hand side of Eq. (23) subtracting from the righthand side of Eq. (23) equals exactly

$$
\begin{aligned}
\sqrt{\ell}[ & \left.-2 \mathbf{W}_{1}+\mathbf{K}_{0}^{-1} \mathbf{R}_{j} \partial_{x_{j}} \mathbf{W}_{1}+\partial_{x_{j}} \mathbf{W}_{1} \mathbf{R}_{j} \mathbf{K}_{0}^{-1}\right] \\
& -2 i \sqrt{\ell} \int\left[e^{i \mathbf{q}^{\dagger} \tilde{\mathbf{x}} / \omega_{1}} \hat{\mathbf{V}}(\mathbf{q}) \mathbf{W}_{2}\left(\mathbf{p}-\frac{\mathbf{q}}{2 \omega_{1}}\right)\right. \\
& \left.-\mathbf{W}_{2}\left(\mathbf{p}-\frac{\mathbf{q}}{2 \omega_{2}}\right) \hat{\mathbf{V}}^{\dagger}(\mathbf{q}) e^{-i \mathbf{q}^{\dagger} \tilde{\mathbf{x}} / \omega_{2}}\right] \mathrm{d} \mathbf{q} \\
& +\ell\left[-2 \mathbf{W}_{2}+\mathbf{K}_{0}^{-1} \mathbf{R}_{j} \partial_{x_{j}} \mathbf{W}_{2}+\partial_{x_{j}} \mathbf{W}_{2} \mathbf{R}_{j} \mathbf{K}_{0}^{-1}\right],
\end{aligned}
$$

which vanishes in a suitable sense as $\ell \rightarrow 0$ [1].

With Eqs. (35), (36), and (38), Eq. (41) is an implicit form of the $2 \mathrm{f}-\mathrm{RT}$ equations that determines the leadingorder coherence matrix. Our next step is to write Eq. (41) explicitly in terms of the spectral densities of the medium fluctuations.

\section{2f-RT EQUATIONS}

Calculation with the left-hand side of Eq. (41) is tedious but straightforward, as it involves only the second-order correlations of $\mathbf{V}$. This is carried out in Appendix B.

To state the full result in a concise form, let us introduce the following quantities. Define the scattering tensors $\mathfrak{S}^{\tau}(\mathbf{p}, \mathbf{q})=\left[\mathcal{S}_{\xi \nu \alpha \zeta}^{\tau}(\mathbf{p}, \mathbf{q})\right]$ as

$$
\mathcal{S}_{\xi \alpha \nu \zeta}^{\tau}(\mathbf{p}, \mathbf{q})=d_{s}^{\tau, \xi^{*}}(\mathbf{p}) e_{i}^{\tau, \alpha}(\mathbf{q}) \Phi_{s i f g}(\omega(\mathbf{p}-\mathbf{q})) d_{f}^{\tau, \nu}(\mathbf{p}) e_{g}^{\tau, \zeta^{*}}(\mathbf{q}) .
$$

Using Eqs. (15)-(18), one can derive the alternative expressions for $\mathcal{S}$ :

$$
\begin{aligned}
\mathcal{S}_{\xi \alpha \nu \zeta}^{\tau}(\mathbf{p}, \mathbf{q}) & =e_{g}^{\tau, \xi^{*}}(\mathbf{p}) d_{f}^{\tau, \alpha}(\mathbf{q}) \Psi_{f g s i}^{*}(\omega(\mathbf{q}-\mathbf{p})) d_{s}^{\tau, \nu}(\mathbf{p}) e_{i}^{\tau, \zeta^{*}}(\mathbf{q}) \\
& =d_{s}^{\tau, \xi^{*}}(\mathbf{p}) e_{i}^{\tau, \alpha}(\mathbf{q}) \Psi_{f g s i}(\omega(\mathbf{q}-\mathbf{p})) e_{g}^{\tau, \nu}(\mathbf{p}) d_{f}^{\tau, \zeta^{*}}(\mathbf{q}) .
\end{aligned}
$$

With Eqs. (15), (16), and (43) it is also straightforward to check that

$$
\mathcal{S}_{\nu \zeta \xi \alpha}^{\tau^{*}}(\mathbf{p}, \mathbf{q})=\mathcal{S}_{\xi \alpha \nu \zeta}^{\tau}(\mathbf{p}, \mathbf{q})=\mathcal{S}_{\zeta \nu \alpha \xi}^{\tau}(\mathbf{q}, \mathbf{p}) .
$$

For any $\mathfrak{M}_{\mathbf{p}}$-valued field $\mathbf{G}(\mathbf{p})$, define the $(\xi, \nu)$ component of the tensor $\mathfrak{S}^{\tau}(\mathbf{p}, \mathbf{q}): \mathbf{G}(\mathbf{q})$ as

$$
\left[\mathfrak{S}^{\tau}(\mathbf{p}, \mathbf{q}): \mathbf{G}(\mathbf{q})\right]_{\xi \nu}=\sum_{\alpha, \zeta} \mathcal{S}_{\xi \alpha \nu \zeta}^{\tau}(\mathbf{p}, \mathbf{q}) G_{\alpha \zeta}(\mathbf{q})
$$

Define the tensors $\Sigma^{\tau}=\left[\Sigma_{\xi \nu}^{\tau}\right]$ analogously to the total scattering cross section as

$$
\begin{aligned}
\boldsymbol{\Sigma}^{\tau}(\mathbf{p})= & \pi \int \delta\left(\Omega^{\tau}(\mathbf{p})-\Omega^{\tau}(\mathbf{q})\right) \mathfrak{S}^{\tau}(\mathbf{p}, \mathbf{q}): \mathbf{I} \mathrm{d} \mathbf{q} \\
& -i \oint-\left(\Omega^{\tau}(\mathbf{p})-\Omega^{\tau}(\mathbf{q})\right)^{-1} \mathfrak{S}^{\tau}(\mathbf{p}, \mathbf{q}): \mathbf{I} \mathrm{d} \mathbf{q} .
\end{aligned}
$$

The $2 \mathrm{f}-\mathrm{RT}$ equation then reads as

$$
\begin{aligned}
\nabla_{\mathbf{p}} \Omega^{\tau} \cdot \nabla_{\mathbf{x}} \overline{\mathbf{W}}^{\tau}= & 2 \pi \omega^{3} \int \delta\left(\Omega^{\tau}(\mathbf{p})-\Omega^{\tau}(\mathbf{q})\right) \\
& \times e^{-i \beta(\mathbf{q}-\mathbf{p})^{\dagger} \mathbf{x}} \mathfrak{S}^{\tau}(\mathbf{p}, \mathbf{q}): \overline{\mathbf{W}}^{\tau}(\mathbf{q}) \mathrm{d} \mathbf{q} \\
& -\omega^{3}\left(\boldsymbol{\Sigma}^{\tau}(\mathbf{p}) \overline{\mathbf{W}}^{\tau}(\mathbf{p})+\overline{\mathbf{W}}^{\tau}(\mathbf{p}) \mathbf{\Sigma}^{\tau \dagger}(\mathbf{p})\right), \quad \forall \tau .
\end{aligned}
$$

Introducing the new quantity

$$
\mathfrak{W}^{\tau}=e^{-i \beta \mathbf{p}^{\dagger} \mathbf{x}} \overline{\mathbf{W}}^{\tau}(\mathbf{p}),
$$

we recast Eq. (46) into the following form:

$$
\begin{aligned}
\nabla_{\mathbf{p}} \Omega^{\tau} \cdot \nabla_{\mathbf{x}} \mathfrak{W}^{\tau}+i \beta \mathbf{p} \cdot \nabla_{\mathbf{p}} \Omega^{\tau} \mathfrak{W}^{\tau} \\
=2 \pi \omega^{3} \int \delta\left(\Omega^{\tau}(\mathbf{p})-\Omega^{\tau}(\mathbf{q})\right) \mathfrak{S}^{\tau}(\mathbf{p}, \mathbf{q}): \mathfrak{W}^{\tau}(\mathbf{q}) \mathrm{d} \mathbf{q} \\
-\omega^{3}\left[\boldsymbol{\Sigma}^{\tau}(\mathbf{p}) \mathfrak{W}^{\tau}(\mathbf{p})+\mathfrak{W}^{\tau}(\mathbf{p}) \Sigma^{\tau^{\dagger}}(\mathbf{p})\right] .
\end{aligned}
$$

This is the Rayleigh-type scaling behavior typical of a random dielectric. The cubic, instead of quartic, power in $\omega$ is due to the appearance of $\omega$ as the scaling factor in the definition of $2 \mathrm{f}-\mathrm{WD}$ [Eq. (19)]. The quartic-in- $\omega$ law is recovered upon replacing $\mathbf{x}$ with $\mathbf{x} / \omega$ on the left-hand side of Eq. (47).

\section{A. Decoupling: Scalar 2f-RT Equation}

Although, in view of Eq. (27), the zero eigenvalue $\Omega^{0}=0$ has multiplicity two in general, the nonzero eigenvalues in media other than the simplest isotropic medium often have multiplicity one, as we shall see in Section 6. This is closely related to the birefringence effect. Under such circumstances, the 2f-RT equations take a much simplified form, which we now state.

Because $\Omega^{j}, j=1,2,3,4$ are simple (multiplicity one), expression (29) reduces to

$$
\overline{\mathbf{W}}(\mathbf{x}, \mathbf{p})=\sum_{\sigma} \bar{W}^{\sigma}(\mathbf{x}, \mathbf{p}) \mathbf{E}^{\sigma}(\mathbf{p}, \mathbf{p}) .
$$

In other words, the coherence matrices become scalars and the different polarization modes decouple. Consequently, Eq. (46) becomes a scalar equation,

$$
\begin{aligned}
\nabla_{\mathbf{p}} \Omega^{\tau} \cdot \nabla_{\mathbf{x}} \bar{W}^{\tau}= & 2 \pi \omega^{3} \int \delta\left(\Omega^{\tau}(\mathbf{p})-\Omega^{\tau}(\mathbf{q})\right) \\
& \times e^{-i \beta(\mathbf{q}-\mathbf{p})^{\dagger} \mathbf{x}_{\mathfrak{S}} \tau}(\mathbf{p}, \mathbf{q}) \bar{W}^{\tau}(\mathbf{q}) \mathrm{d} \mathbf{q} \\
& -2 \omega^{3} \Sigma^{\tau}(\mathbf{p}) \bar{W}^{\tau}(\mathbf{p}), \quad \forall \tau,
\end{aligned}
$$

where

$$
\mathfrak{S}^{\tau}(\mathbf{p}, \mathbf{q})=d_{s}^{\tau^{*}}(\mathbf{p}) e_{i}^{\tau}(\mathbf{q}) \Phi_{s i f g}(\omega(\mathbf{p}-\mathbf{q})) d_{f}^{\tau}(\mathbf{p}) e_{g}^{\tau^{*}}(\mathbf{q}),
$$




$$
\Sigma^{\tau}(\mathbf{p})=\pi \int \delta\left(\Omega^{\tau}(\mathbf{p})-\Omega^{\tau}(\mathbf{q})\right) \mathfrak{S}^{\tau}(\mathbf{p}, \mathbf{q}) \mathrm{d} \mathbf{q} .
$$

Note that the Cauchy singular integral term in Eq. (50) disappears whenever $\Sigma^{\tau}$ and $\overline{\mathbf{W}}$ commute as in the scalar case. From Eq. (48) we can derive the scalar equation for the quantity $\mathfrak{W}^{\tau}=e^{-i \beta \mathbf{p}^{\dagger} \mathbf{x}} \bar{W}^{\tau}(\mathbf{p})$ as before.

\section{SPECIAL MEDIA}

In this section, we consider the eigenstructure of the dispersion matrix $\mathbf{K}_{0}^{-1} p_{j} \Re_{j}$ associated with the various background media for which the scattering tensor can be computed explicitly. Throughout Subsections 6.B, 6.C, and $6 . \mathrm{D}$, the symbol $\times$ stands for the cross (vector) product.

\section{A. Isotropic Medium}

In the simplest case of an isotropic medium, there are two nonzero eigenvalues: $\Omega^{+}(\mathbf{p})=c_{0}|\mathbf{p}|, \Omega^{-}(\mathbf{p})=-c_{0}|\mathbf{p}|$, each of multiplicity two. Let $\hat{\mathbf{p}}=\mathbf{p} /|\mathbf{p}|$ and let $\hat{\mathbf{p}}_{\perp}^{+}, \hat{\mathbf{p}}_{\perp}^{-}$be any pair of unit vectors orthogonal to each other and to $\hat{\mathbf{p}}$ so that $\left\{\hat{\mathbf{p}}_{\perp}^{+}, \hat{\mathbf{p}}_{\perp}^{-}, \hat{\mathbf{p}}\right\}$ form a right-handed coordinate frame. Let $\left\{\hat{\mathbf{q}}_{\perp}^{+}, \hat{\mathbf{q}}_{\perp}^{-}, \hat{\mathbf{q}}\right\}$ be similarly defined. The eigenvectors are

$$
\begin{array}{cc}
\mathbf{e}^{+,+}(\mathbf{p})=\left(\begin{array}{c}
\frac{1}{\sqrt{2 \epsilon_{0}}} \hat{\mathbf{p}}_{\perp}^{+} \\
\frac{1}{\sqrt{2 \mu_{0}}} \hat{\mathbf{p}}_{\perp}^{-}
\end{array}\right), \quad \mathbf{e}^{+,-}(\mathbf{p})=\left(\begin{array}{c}
\frac{1}{\sqrt{2 \epsilon_{0}}} \hat{\mathbf{p}}_{\perp}^{-} \\
-\frac{1}{\sqrt{2 \mu_{0}}} \hat{\mathbf{p}}_{\perp}^{+}
\end{array}\right), \\
\mathbf{e}^{-,+}(\mathbf{p})=\left(\begin{array}{c}
\frac{1}{\sqrt{2 \epsilon_{0}}} \hat{\mathbf{p}}_{\perp}^{+} \\
-\frac{1}{\sqrt{2 \mu_{0}}} \hat{\mathbf{p}}_{\perp}^{-}
\end{array}\right), \quad \mathbf{e}^{-,-}(\mathbf{p})=\left(\begin{array}{c}
\frac{1}{\sqrt{2 \epsilon_{0}}} \hat{\mathbf{p}}_{\perp}^{-} \\
\frac{1}{\sqrt{2 \mu_{0}}} \hat{\mathbf{p}}_{\perp}^{+}
\end{array}\right) .
\end{array}
$$

Denote the spectral densities of $\tilde{\epsilon}$ and $\tilde{\mu}$ by $\Phi_{\epsilon}$ and $\Phi_{\mu}$, respectively, and denote the cross spectral densities by $\Phi_{\epsilon \mu}, \Phi_{\mu \epsilon}$. We have $\mathfrak{S}^{\tau}=\left[\mathcal{S}_{\xi \nu \alpha \zeta}^{\tau}\right]$ with

$$
\begin{aligned}
\mathcal{S}_{\xi \alpha \nu \zeta}^{\tau}(\mathbf{p}, \mathbf{q})= & \frac{1}{4}\left[\Phi_{\epsilon}(\omega(\mathbf{p}-\mathbf{q})) \hat{\mathbf{p}}_{\perp}^{\xi \dagger} \hat{\mathbf{q}}_{\perp}^{\alpha} \hat{\mathbf{q}}_{\perp}^{\zeta \dagger} \hat{\mathbf{p}}_{\perp}^{\nu}\right. \\
& -\Phi_{\epsilon \mu}(\omega(\mathbf{p}-\mathbf{q})) \hat{\mathbf{p}}_{\perp}^{\xi \dagger} \hat{\mathbf{q}}_{\perp}^{\alpha} \hat{\mathbf{q}}_{\perp}^{-\zeta \dagger} \hat{\mathbf{p}}_{\perp}^{-\nu} \\
& -\Phi_{\mu \epsilon}(\omega(\mathbf{p}-\mathbf{q})) \hat{\mathbf{p}}_{\perp}^{-\xi^{\dagger}} \hat{\mathbf{q}}_{\perp}^{-\alpha} \hat{\mathbf{q}}_{\perp}^{\zeta \dagger} \hat{\mathbf{p}}_{\perp}^{\nu} \\
& \left.+\Phi_{\mu}(\omega(\mathbf{p}-\mathbf{q})) \hat{\mathbf{p}}_{\perp}^{-\xi^{\dagger}} \hat{\mathbf{q}}_{\perp}^{-\alpha} \hat{\mathbf{q}}_{\perp}^{-\zeta \dagger} \hat{\mathbf{p}}_{\perp}^{-\nu}\right]
\end{aligned}
$$

for $\tau, \xi, \alpha, \zeta, \nu= \pm$. Equation (46) can now be written as

$$
\begin{aligned}
c_{0} \hat{\mathbf{p}} \cdot \nabla_{\mathbf{x}} \overline{\mathbf{W}}^{ \pm}= & \pm \frac{\pi \omega^{3}|\mathbf{p}|^{2}}{4 c_{0}}\left[2 \int e^{-i \beta(\mathbf{q}-\mathbf{p})^{\dagger} \mathbf{x}} \delta(|\mathbf{p}|-|\mathbf{q}|)\right. \\
& \times \mathfrak{S}^{ \pm}(\mathbf{p}, \mathbf{q}): \overline{\mathbf{W}}^{ \pm}(\mathbf{q}) \mathrm{d} \hat{\mathbf{q}} \\
& -\int \delta(|\mathbf{p}|-|\mathbf{q}|) \mathfrak{S}^{ \pm}(\mathbf{p}, \mathbf{q}): \mathbf{I} \mathrm{d} \hat{\mathbf{q}} \overline{\mathbf{W}}^{ \pm} \\
& \left.-\overline{\mathbf{W}}^{ \pm} \int \delta(|\mathbf{p}|-|\mathbf{q}|) \mathfrak{S}^{ \pm}(\mathbf{p}, \mathbf{q}): \mathbf{I} \mathrm{d} \hat{\mathbf{q}}\right] .
\end{aligned}
$$

Property (44) and expression (51) imply that $\mathcal{S}_{\xi \alpha \nu \zeta}^{\tau}(\mathbf{p}, \mathbf{q})$ $=\mathcal{S}_{\xi \alpha \nu \zeta}^{\tau}(\mathbf{q}, \mathbf{p})$ and hence the Cauchy principal value term in Eq. (45) disappears.

Often, in a scattering atmosphere for instance, $\tilde{\mu}=0$ is a good approximation and in such cases the only nonzero term in the scattering kernel is

$$
\mathcal{S}_{\xi \alpha \nu \zeta}^{\tau}(\mathbf{p}, \mathbf{q})=\frac{1}{4} \Phi_{\epsilon}(\omega(\mathbf{p}-\mathbf{q})) \hat{\mathbf{p}}_{\perp}^{\xi \dagger} \hat{\mathbf{q}}_{\perp}^{\alpha} \hat{\mathbf{q}}_{\perp}^{\zeta \dagger} \hat{\mathbf{p}}_{\perp}^{\nu}
$$

This is the setting for which Chandrasekhar originally derived his famous equation of transfer [20], and Eq. (52) is just the two-frequency version of Chandrasekhar's equation.

In the same setting, the new features in Eq. (52) beyond Chandrasekhar's transfer equation are the frequency shift $\beta$ and the general form of the power spectrum $\Phi_{\epsilon}$. In Chandrasekhar's and other cases, the medium consists of randomly distributed particles of size smaller than the wavelength $[3,21]$. Such a discrete medium corresponds to a random field $\mathbf{V}$ that is a sum of $\delta$-like functions randomly distributed according to the Poisson point process whose spectral density tensor $\boldsymbol{\Phi}$ can be calculated.

\section{B. Chiral Medium}

A chiral medium is a reciprocal, biisotropic medium with the constitutive matrix

$$
\mathbf{K}_{0}=\left[\begin{array}{cc}
\epsilon_{0} \mathbf{I} & i \chi \mathbf{I} \\
-i \chi \mathbf{I} & \mu_{0} \mathbf{I}
\end{array}\right]
$$

where $\chi \in \mathbb{R}$ is the magnetoelectric coefficient. To maintain a positive-definite $\mathbf{K}_{0}$ we assume $\chi^{2}<\epsilon_{0} \mu_{0}$. We then have

$$
\mathbf{K}_{0}^{-1} p_{j} \mathbf{R}_{j}=\frac{c_{0}}{1-\kappa^{2}}\left[\begin{array}{cc}
z \mathbf{I} & -i \kappa \mathbf{I} \\
i \kappa \mathbf{I} & z^{-1} \mathbf{I}
\end{array}\right]\left[\begin{array}{cc}
0 & -\mathbf{p} \times \\
\mathbf{p} \times & 0
\end{array}\right]
$$

where $z=\sqrt{\mu_{0} / \epsilon_{0}}>0$ is the impedance and $\kappa=\chi c_{0}$ is the chirality parameter. The four nonzero simple eigenvalues are $\Omega^{1}=c_{0}|\mathbf{p}|(1+\kappa)^{-1}, \Omega^{2}=c_{0}|\mathbf{p}|(1-\kappa)^{-1}, \Omega^{3}=c_{0}|\mathbf{p}|(\kappa-1)^{-1}$, $\Omega^{4}=c_{0}|\mathbf{p}|(-\kappa-1)^{-1}$ and their corresponding eigenvectors are

$$
\begin{array}{ll}
\mathbf{e}^{1} \sim\left(\begin{array}{cc}
-i \hat{\mathbf{p}}_{\perp}^{1}+\hat{\mathbf{p}}_{\perp}^{2} \\
-\frac{\hat{\mathbf{p}}_{\perp}^{1}}{z}-i \frac{\hat{\mathbf{p}}_{\perp}^{2}}{z}
\end{array}\right), \quad \mathbf{e}^{2} \sim\left(\begin{array}{c}
i \hat{\mathbf{p}}_{\perp}^{1}+\hat{\mathbf{p}}_{\perp}^{2} \\
-\frac{\hat{\mathbf{p}}_{\perp}^{1}}{z}+i \frac{\hat{\mathbf{p}}_{\perp}^{2}}{z}
\end{array}\right), \\
\mathbf{e}^{3} \sim\left(\begin{array}{c}
-i \hat{\mathbf{p}}_{\perp}^{1}+\hat{\mathbf{p}}_{\perp}^{2} \\
\frac{\hat{\mathbf{p}}_{\perp}^{1}}{z}+i \frac{\hat{\mathbf{p}}_{\perp}^{2}}{z}
\end{array}\right), & \mathbf{e}^{4} \sim\left(\begin{array}{l}
i \hat{\mathbf{p}}_{\perp}^{1}+\hat{\mathbf{p}}_{\perp}^{2} \\
\frac{\hat{\mathbf{p}}_{\perp}^{1}}{z}-i \frac{\hat{\mathbf{p}}_{\perp}^{2}}{z}
\end{array}\right),
\end{array}
$$

where $\hat{\mathbf{p}}_{\perp}^{1}=\hat{\mathbf{p}}_{\perp}^{+}, \hat{\mathbf{p}}_{\perp}^{2}=\hat{\mathbf{p}}_{\perp}^{-}$. Note also that $\Omega^{4}=-\Omega^{1}, \Omega^{3}=-\Omega^{2}$. As $|\kappa|<1$ (since $\chi^{2}<\epsilon_{0} \mu_{0}$ ), $\mathbf{e}^{1}, \mathbf{e}^{2}$ are the forwardpropagating modes and $\mathbf{e}^{3}, \mathbf{e}^{4}$ the backward-propagating modes.

For the medium fluctuation $\mathbf{V}$ we may use the biisotropy form 


$$
\left[\begin{array}{cc}
a \mathbf{I} & i \mu_{0} b \mathbf{I} \\
-i \epsilon_{0} b \mathbf{I} & a \mathbf{I}
\end{array}\right],
$$

where $a, b \in \mathbb{R}$ are stationary random functions of $\mathbf{x}$ with power spectral densities $\Phi_{a}, \Phi_{b}$ and cross-spectral density $\Phi_{a b}$. This particular form is derived from the commutativity relation (10).

The splitting into two distinct positive dispersion relations is a case of birefrigence where two distinct phase velocities, $c_{0} /(1 \pm \kappa)$, arise depending on the polarization. As discussed in Subsection 5.A, due to the birefringence the chiral medium does not depolarize the electromagnetic waves. For the sake of space, we leave to the reader to work out the scattering tensor from Eqs. (49) and (50).

\section{Birefrigence in Anisotropic Crystals}

Generally speaking, an anisotropic medium permits two monochromatic plane waves with two different linear polarizations and two different velocities to propagate in any given direction [10]. This again gives rise to the birefringence effect.

The only optically isotropic crystal is the cubic crystal. All the other types of crystals are optically anisotropic in general. In the system of principal dielectric axes, the permitivity-permeability tensor of a crystal, which is always a real, symmetric matrix, can be diagonalized as $\mathbf{K}_{0}=\operatorname{diag}\left[\epsilon_{x}, \epsilon_{y}, \epsilon_{z}, 1,1,1\right]$. One type of anisotropic crystal is the uniaxial crystal for which $\epsilon_{x}=\epsilon_{y}=\epsilon_{\perp} \neq \epsilon_{z}=\epsilon_{\|}$(if the distinguished direction, the optic axis, is taken as the $z$ axis). There exist two distinct dispersion relations for the forward modes:

$$
\Omega^{o}=\frac{|\mathbf{p}|}{\sqrt{\epsilon_{\perp}}}, \quad \Omega^{e}=\sqrt{\frac{p_{3}^{2}}{\epsilon_{\perp}}+\frac{p_{1}^{2}+p_{2}^{2}}{\epsilon_{\|}}} .
$$

The backward modes correspond to $-\Omega^{0},-\Omega^{e}$. The corresponding wave-vector surface consists of a sphere and an ovaloid, a surface of revolution. $\Omega^{o}$ corresponds to the ordinary wave with a velocity independent of the wavevector, and $\Omega^{e}$ corresponds to the extraordinary wave with a velocity depending on the angle between the wave vector and the optic axis [10].

Let $\mathbf{d}^{o}, \mathbf{d}^{e}$ be the associated left eigenvectors. Set $\mathbf{K}_{0}^{\epsilon}$ $=\operatorname{diag}\left[\epsilon_{\perp}, \epsilon_{\perp}, \epsilon_{\|}\right]$and let $\mathbf{a}^{\sigma}$ solve the following symmetric eigenvalue problem:

$$
-\mathbf{p} \times\left(\mathbf{K}_{0}^{\epsilon}\right)^{-1} \mathbf{p} \times \mathbf{a}^{\sigma}=\left(\Omega^{\sigma}\right)^{2} \mathbf{a}^{\sigma}, \quad \sigma=e, o .
$$

Then the left eigenvectors $\mathbf{d}^{\sigma}$ can be written as

$$
\mathbf{d}^{\sigma} \sim\left(\begin{array}{c}
-\mathbf{p} \times \mathbf{a}^{\sigma} \\
\Omega^{\sigma} \mathbf{a}^{\sigma}
\end{array}\right), \quad \sigma=e, o .
$$

The same formula applies to the backward modes. Equation (55) has the following solutions:

$$
\mathbf{a}^{e}=\left(-p_{2}, p_{1}, 0\right)^{\dagger}, \quad \mathbf{a}^{o}=\left(p_{1}, p_{2},-\frac{p_{1}^{2}+p_{2}^{2}}{p_{3}}\right)^{\dagger},
$$

from which we see that the wave is linearly polarized.

The other type of anisotropic crystal is the biaxial crystal for which there are also two distinct, but more complicated, dispersion relations, both associated with the ex- traordinary waves [10]. In contrast, the two distinct dispersion relations of a chiral medium give rise to two ordinary waves, as the two-wave-vector surface consists of two concentric spheres centered at $\mathbf{p}=0$.

It should be emphasized that a plane wave propagating in an anisotropic crystal is linearly polarized in certain planes, whereas a plane wave propagating in the isotropic medium is in general elliptically polarized and is linearly polarized only in particular cases. In the anisotropic as well as the chiral media, the different polarizations decouple in the RT equations, and the depolarization effect is absent.

\section{Gyrotropic Medium}

In the presence of a static external magnetic field $\mathbf{H}_{\text {ext }}$, the permittivity tensor $\mathbf{K}_{0}^{\epsilon}$ is no longer symmetrical; it is generally a complex Hermitian matrix. Here we consider the simplest such constitutive relation,

$$
\mathbf{D}=\epsilon_{0} \mathbf{E}-i \mathbf{g} \times \mathbf{E}, \quad \mathbf{B}=\mathbf{H},
$$

where $\mathbf{g}=f \mathbf{H}_{\text {ext }}, f \in \mathbb{R}$, is the gyration vector. Equivalently, we can write

$$
\mathbf{E}=\frac{1}{\epsilon_{0}^{2}-|\mathbf{g}|^{2}}\left(\epsilon_{0} \mathbf{D}+i \mathbf{g} \times \mathbf{D}-\frac{1}{\epsilon_{0}} \mathbf{g g}^{\dagger} \mathbf{D}\right) .
$$

In this case there are two distinct forward dispersion relations [9]

$$
\Omega^{1}=c_{0}\left|\mathbf{p}+\frac{\Omega^{1}}{2} \mathbf{g}\right|, \quad \Omega^{2}=c_{0}\left|\mathbf{p}-\frac{\Omega^{2}}{2} \mathbf{g}\right|,
$$

where $c_{0}=1 / \sqrt{\epsilon_{0}}$. Clearly the wave-vector surface consists of two spheres of the same radius but different centers. This should be contrasted with the case of chiral media for which the wave-vector surface consists of two concentric spheres of different radii.

The associated (left) eigenvectors $\mathbf{d}^{\sigma}, \sigma=1,2$ can be written as in Eq. (56) with $\mathbf{a}^{\sigma}$ solving Eq. (55) and $\mathbf{K}_{0}^{\epsilon}$ corresponding to Eq. (57). Let $\mathbf{g}=g_{1} \hat{\mathbf{p}}_{\perp}^{1}+g_{2} \hat{\mathbf{p}}_{\perp}^{2}+g_{3} \hat{\mathbf{p}}$. We can write the three-dimensional vector $\mathbf{a}^{\sigma}$ as $\mathbf{a}^{\sigma}=\hat{\mathbf{p}}_{\perp}^{1}+\gamma_{\sigma} \hat{\mathbf{p}}_{\perp}^{2}$ with

$$
\gamma_{\sigma}=\frac{g_{2}^{2}-g_{1}^{2}-(-1)^{\sigma} \sqrt{\left(g_{1}^{2}+g_{2}^{2}\right)^{2}+4 \epsilon_{0}^{2} g_{3}^{2}}}{2\left(g_{1} g_{2}-i \epsilon_{0} g_{3}\right)}, \quad \sigma=1,2 .
$$

We see that the wave is in general elliptically polarized or linearly polarized when $\mathbf{g}$ is orthogonal to the wave vector $\mathbf{p}$ and circularly polarized when $\mathbf{g}$ is parallel to $\mathbf{p}$. Again, the simplicity of the eigenvalues implies that depolarization is absent in the gyrotropic media.

\section{GEOMETRICAL 2f-RT}

We have seen in Subsection 5.A how a scalar 2f-RT equation naturally arises in a birefringent medium. In this section, we show that a scalar $2 \mathrm{f}-\mathrm{RT}$ equation can also arise in a depolarizing medium such as the isotropic medium discussed in Subsection 6.A. Depolarization can mix different polarization modes and result in scalar-like coherence matrices $\overline{\mathbf{W}}^{\tau} \approx \bar{W} \tau \mathbf{T}, \tau= \pm$ (see Subsection 6.A for notation). The other purpose of this section is to show 
smooth transition from Eq. (46) to the Fokker-Planck equation, previously derived for the scalar waves [1,7], in geometrical optics through rapid depolarization.

Let us start with the general setting and replace $\boldsymbol{\Phi}(\cdot)$ in Eq. (47) with $\gamma^{-4} \boldsymbol{\Phi}(\cdot / \gamma)$, where the small parameter $\gamma$ is roughly the ratio of the wavelength to the correlation length of the medium fluctuations. In other words, we consider the geometrical optics regime. The quartic power in $\gamma$ is indicative of Rayleigh-type scattering. Consider the change of variable $\mathbf{q}=\mathbf{p}+\gamma \mathbf{k}$ in the scattering term of Eq. (47). With this and the ansatz $\mathfrak{W}^{\tau}=e^{-i \beta \mathbf{p} \dagger \mathbf{x}} \bar{W}^{\tau}$, the scattering term becomes approximately

$$
\begin{aligned}
2 \pi \omega^{3} \gamma^{-1} \int \mathrm{d} \mathbf{k} \delta\left(\Omega^{\tau}(\mathbf{p}+\gamma \mathbf{k})-\Omega^{\tau}(\mathbf{p})\right) d_{s}^{\tau, \xi^{*}}(\mathbf{p}) d_{f}^{\tau, \nu}(\mathbf{p}) \\
\quad \times \Phi_{\text {sifg }}(\omega \mathbf{k}) \sum_{\alpha} e_{i}^{\tau, \alpha}(\mathbf{p}+\gamma \mathbf{k}) e_{g}^{\tau, \alpha^{*}}(\mathbf{p}+\gamma \mathbf{k}) \\
\quad \times\left[\mathfrak{W}^{\tau}(\mathbf{p})+\gamma \mathbf{k} \cdot \nabla_{\mathbf{p}} \mathfrak{W}^{\tau}(\mathbf{p})+\frac{\gamma^{2}}{2} k_{l} k_{j} \partial_{p_{l}} \partial_{p_{j}} \mathfrak{W}^{\tau}(\mathbf{p})\right] .
\end{aligned}
$$

The first term in Eq. (58) cancels exactly with $\Sigma^{\tau}(\mathbf{p}) \mathfrak{W}^{\tau}(\mathbf{p})+\mathfrak{W}^{\tau}(\mathbf{p}) \boldsymbol{\Sigma}^{\tau \dagger}(\mathbf{p})$ on the right-hand side of Eq. (47). The second term in Eq. (58) yields the first-order differential operator

$$
\begin{aligned}
& {\left[\pi \omega^{3} d_{s}^{\tau, \xi^{*}} d_{f}^{\tau, \nu} \sum_{\alpha} e_{i}^{\tau, \alpha} e_{g}^{\tau, \alpha^{*}}\left[\partial_{p_{l}} \partial_{p_{j}} \Omega^{\tau}\right]\right.} \\
& \times \int k_{l} k_{j} \delta^{\prime}\left(\mathbf{k} \cdot \nabla_{\mathbf{p}} \Omega^{\tau}\right) \Phi_{\text {sifg }}(\omega \mathbf{k}) \mathbf{k} \mathrm{d} \mathbf{k} \\
& +2 \pi \omega^{3} d_{s}^{\tau, \xi^{*}} d_{f}^{\tau, \nu} \sum_{\alpha} \partial_{p_{l}}\left[e_{i}^{\tau, \alpha} e_{g}^{\tau, \alpha^{*}}\right] \\
& \left.\times \int k_{l} \delta\left(\mathbf{k} \cdot \nabla_{\mathbf{p}} \Omega^{\tau}\right) \Phi_{\text {sifg }}(\omega \mathbf{k}) \mathbf{k} \mathrm{d} \mathbf{k}\right] \cdot \nabla_{\mathbf{p} \mathfrak{W}^{\tau}}
\end{aligned}
$$

where $\delta^{\prime}$ is the derivative of the Dirac delta function. The third term in Eq. (58) yields the second-order differential operator

$$
\begin{gathered}
\pi \omega^{3} d_{s}^{\tau, \xi^{*}} d_{f}^{\tau, \nu} \sum_{\alpha} e_{i}^{\tau, \alpha} e_{g}^{\tau, \alpha^{*}} \int \delta\left(\mathbf{k} \cdot \nabla_{\mathbf{p}} \Omega^{\tau}\right) \\
\times \Phi_{\text {sifg }}(\omega \mathbf{k}) k_{m} k_{n} \mathrm{~d} \mathbf{k} \partial_{p_{m}} \partial_{p_{n}} \mathfrak{W}^{\tau}
\end{gathered}
$$

In order to match the left-hand side of Eq. (47), which is a scalar in the case of complete depolarization, Eq. (60) and each term in Eq. (59) must be proportional to $\delta_{\xi, \nu}$ as well. This happens, for instance, for the isotropic medium with Eq. (53). In this case,

$$
\begin{aligned}
& (59)=\pi \delta_{\xi, \nu} \frac{\omega^{3}}{4} \int \mathbf{k} \cdot \nabla_{\mathbf{p}}(\mathbf{k} \cdot \hat{\mathbf{p}}) \delta^{\prime}(\mathbf{k} \cdot \hat{\mathbf{p}}) \Phi_{\epsilon}(\omega \mathbf{k}) \mathbf{k} \mathrm{d} \mathbf{k} \cdot \nabla_{\mathbf{p}} \mathfrak{W}^{\tau}, \\
& (60)=\pi \delta_{\xi, \nu} \frac{\omega^{3}}{4 c_{0}} \int \delta(\mathbf{k} \cdot \hat{\mathbf{p}}) \Phi_{\epsilon}(\omega \mathbf{k}) k_{m} k_{n} \mathrm{~d} \mathbf{k} \partial_{p_{m}} \partial_{p_{n}} \mathfrak{W}^{\tau},
\end{aligned}
$$

and hence the $2 \mathrm{f}-\mathrm{RT}$ equation (47) becomes

$$
\pm c_{0} \hat{\mathbf{p}} \cdot \nabla_{\mathbf{x}} \mathfrak{W}^{ \pm} \pm i \beta c_{0}|\mathbf{p}| \mathfrak{W}^{ \pm}=\nabla_{\mathbf{p}} \cdot \mathbf{D} \nabla_{\mathbf{p}} \mathfrak{W}^{ \pm}
$$

with the diffusion coefficient

$$
\mathbf{D}=\frac{\pi \omega^{3}}{4 c_{0}} \int \delta(\mathbf{k} \cdot \hat{\mathbf{p}}) \Phi_{\epsilon}(\omega \mathbf{k}) \mathbf{k} \mathbf{k}^{\dagger} \mathrm{d} \mathbf{k}
$$

which is the same Fokker-Planck-type equation derived by a rigorous, probabilistic method from the geometrical optics of the scalar wave previously [6].

Applying the same procedure to the scalar 2f-RT equation for the quantity $\mathfrak{W}^{\tau}$ of the birefringence case discussed in Subsection 5.A, we obtain

$$
\nabla_{\mathbf{p}} \Omega^{\tau} \cdot \nabla_{\mathbf{x}} \mathfrak{W}^{\tau}+i \beta \mathbf{p} \cdot \nabla_{\mathbf{p}} \Omega^{\tau} \mathfrak{W}^{\tau}=\nabla_{\mathbf{p}} \cdot \mathbf{D} \nabla_{\mathbf{p}} \mathfrak{W}^{\tau}
$$

where the diffusion coefficient $\mathbf{D}$ is given by

$$
\begin{aligned}
\mathbf{D}(\mathbf{p})= & \pi \omega^{3} d_{s}^{\tau^{*}}(\mathbf{p}) e_{i}^{\tau}(\mathbf{p}) d_{f}^{\tau}(\mathbf{p}) e_{g}^{\tau^{*}}(\mathbf{p}) \\
& \times \int \delta(\mathbf{k} \cdot \hat{\mathbf{p}}) \Phi_{s i f g}(\omega \mathbf{k}) \mathbf{k} \mathbf{k}^{\dagger} \mathrm{d} \mathbf{k} .
\end{aligned}
$$

\section{CONCLUSION}

Starting with the symmetrical Wigner-Moyal equation (23), we have systematically derived the $2 \mathrm{f}-\mathrm{RT}$ equations (46)-(48) in the radiative transfer regime characterized by weak-coupling scaling (9). The main assumptions about the medium are that the background is uniform and has a either positive or negative definite constitutive matrix and that the fluctuations are zero-mean statistically homogeneous processes.

We now turn to the antisymmetrical Wigner-Moyal equation (24) and discuss the consequence of its leadingorder terms, which are

$$
2 \overline{\mathbf{W}}=\mathbf{K}_{0}^{-1} p_{j} \mathbf{R}_{j} \overline{\mathbf{W}}+\overline{\mathbf{W}} p_{j} \mathbf{R}_{j} \mathbf{K}_{0}^{-1} .
$$

In view of Eq. (29) this is equivalent to $1=\Omega^{\sigma}(\mathbf{p})$. Note again that the variable $\mathbf{p}$ has the dimension of inverse velocity. Therefore the two-space-time correlations of the time-dependent polarized wave field $\mathbf{u}$ are given approximately by

$$
\begin{aligned}
& \left\langle\mathbf{u}\left(t_{1}, \mathbf{x}_{1}\right) \mathbf{u}^{\dagger}\left(t_{2}, \mathbf{x}_{2}\right)\right\rangle \\
& \sim \sum_{\tau, \alpha \zeta} \iint e^{i \omega \beta t} e^{-i \omega \tau / \ell} \int_{\Omega^{\sigma}(\mathbf{p})=1} e^{i \mathbf{p}^{\dagger}(\mathbf{y}+\beta \mathbf{x})} \mathfrak{W}_{\alpha \zeta}^{\tau}(\mathbf{x}, \mathbf{p}) \\
& \quad \times \mathbf{E}^{\tau, \alpha \zeta}(\mathbf{p}, \mathbf{p}) \mathrm{d} \Omega(\mathbf{p}) \mathrm{d} \omega \mathrm{d} \beta,
\end{aligned}
$$

with $\mathbf{x}=\omega\left(\mathbf{x}_{1}+\mathbf{x}_{2}\right) / 2, \mathbf{y}=\omega\left(\mathbf{x}_{1}-\mathbf{x}_{2}\right) / \ell$, where $\mathfrak{W}^{\tau}=\left[\mathfrak{W}_{\alpha \zeta}^{\tau}\right]$ is the solution to Eq. (47) and $d \Omega(\mathbf{p})$ is the area element of the surface $\Omega^{\sigma}(\mathbf{p})=1$.

Parallel to the case of scalar waves, one can also work out the implications of the polarization on the problems of imaging and time-reversal communications, as discussed in the Introduction and references therein, from the $2 \mathrm{f}-\mathrm{RT}$ theory developed here. 


\section{APPENDIX A: DERIVATION OF THE WIGNER-MOYAL EQUATION}

Applying the operator $\mathbf{R}_{j} \partial / \partial x_{j}$ to $\mathbf{W}$ we have

$$
\begin{aligned}
\mathbf{R}_{j} \frac{\partial}{\partial x_{j}} \mathbf{W}(\mathbf{x}, \mathbf{p})= & \frac{1}{(2 \pi)^{3}} \int e^{-i \mathbf{p}^{\dagger} \mathbf{y}} \mathbf{R}_{j} \frac{\partial}{\partial x_{j}} \mathbf{U}_{1}\left(\frac{\mathbf{x}}{\omega_{1}}+\frac{\mathbf{y}}{2 \omega_{1}}\right) \\
& \times \mathbf{U}_{2}^{\dagger}\left(\frac{\mathbf{x}}{\omega_{2}}-\frac{\mathbf{y}}{2 \omega_{2}}\right) \mathrm{d} \mathbf{y}+\frac{1}{(2 \pi)^{3}} \int e^{-i \mathbf{p}^{\dagger} \mathbf{y} \mathbf{R}_{j}} \\
& \times \mathbf{U}_{1}\left(\frac{\mathbf{x}}{\omega_{1}}+\frac{\mathbf{y}}{2 \omega_{1}}\right) \frac{\partial}{\partial x_{j}} \mathbf{U}_{2}^{\dagger}\left(\frac{\mathbf{x}}{\omega_{2}}-\frac{\mathbf{y}}{2 \omega_{2}}\right) \mathrm{d} \mathbf{y}, \\
= & \frac{1}{(2 \pi)^{3}} \int e^{-i \mathbf{p}^{\dagger} \mathbf{y}} \omega_{1}^{-1} \mathbf{R}_{j} \frac{\partial}{\partial x_{j}} \mathbf{U}_{1}\left(\frac{\mathbf{x}}{\omega_{1}}+\frac{\mathbf{y}}{2 \omega_{1}}\right) \\
& \times \mathbf{U}_{2}^{\dagger}\left(\frac{\mathbf{x}}{\omega_{2}}-\frac{\mathbf{y}}{2 \omega_{2}}\right) \mathrm{d} \mathbf{y}-\frac{2}{(2 \pi)^{3}} \int e^{-i \mathbf{p}^{\dagger} \mathbf{y} \mathbf{R}_{j}} \\
& \times \mathbf{U}_{1}\left(\frac{\mathbf{x}}{\omega_{1}}+\frac{\mathbf{y}}{2 \omega_{1}}\right) \frac{\partial}{\partial y_{j}} \mathbf{U}_{2}^{\dagger}\left(\frac{\mathbf{x}}{\omega_{2}}-\frac{\mathbf{y}}{2 \omega_{2}}\right) \mathrm{d} \mathbf{y} .
\end{aligned}
$$

Integrating by parts with the second integral and using (6) we obtain

$$
\begin{aligned}
\mathbf{R}_{j} \frac{\partial}{\partial x_{j}} \mathbf{W}(\mathbf{x}, \mathbf{p})= & \frac{2 i}{(2 \pi)^{3}} \int e^{-i \mathbf{p}^{\dagger} \mathbf{y}} \mathbf{K}\left(\frac{\mathbf{x}}{\omega_{1}}+\frac{\mathbf{y}}{2 \omega_{2}}\right) \mathbf{U}_{1}\left(\frac{\mathbf{x}}{\omega_{1}}+\frac{\mathbf{y}}{2 \omega_{1}}\right) \\
& \times \mathbf{U}_{2}^{\dagger}\left(\frac{\mathbf{x}}{\omega_{2}}-\frac{\mathbf{y}}{2 \omega_{2}}\right) \mathrm{d} \mathbf{y}-\frac{2 i}{(2 \pi)^{3}} p_{j} \mathbf{R}_{j} \\
& \times \int e^{-i \mathbf{p}^{\dagger} \mathbf{y}} \mathbf{U}_{1}\left(\frac{\mathbf{x}}{\omega_{1}}+\frac{\mathbf{y}}{2 \omega_{1}}\right) \mathbf{U}_{2}^{\dagger}\left(\frac{\mathbf{x}}{\omega_{2}}-\frac{\mathbf{y}}{2 \omega_{2}}\right) \mathrm{d} \mathbf{y} .
\end{aligned}
$$

Inserting the spectral representation of $\mathbf{K}$ into the equation and using definition (19), we then obtain Eq. (7).

\section{APPENDIX B: CALCULATION OF EQ. (41)}

\section{Propagation Terms}

We first show that

$$
\operatorname{Tr}\left[\mathbf{D}^{\tau, \xi \nu^{\dagger}}\left(\mathbf{K}_{0}^{-1} \mathbf{R}_{j} \partial_{x_{j}} \overline{\mathbf{W}}+\partial_{x_{j}} \overline{\mathbf{W}} \mathbf{R}_{j} \mathbf{K}_{0}^{-1}\right)\right]=2 \nabla_{\mathbf{p}} \Omega^{\tau} \cdot \nabla_{\mathbf{x}} \bar{W}_{\xi \nu}^{\tau} .
$$

Consider the following calculation:

$$
\begin{aligned}
\mathbf{K}_{0}^{-1} \mathbf{R}_{j} \partial_{x_{j}} \overline{\mathbf{W}}= & \nabla_{\mathbf{p}}\left[\mathbf{K}_{0}^{-1} p_{j} \mathbf{R}_{j}\right] \cdot\left[\nabla_{\mathbf{x}} \bar{W}_{\alpha \zeta}^{\sigma}\right] \mathbf{e}^{\sigma, \alpha} \mathbf{e}^{\sigma, \zeta^{\dagger}} \\
= & \nabla_{\mathbf{p}}\left[\mathbf{K}_{0}^{-1} p_{j} \mathbf{R}_{j} \mathbf{e}^{\sigma, \alpha}\right] \cdot\left[\nabla_{\mathbf{x}} \bar{W}_{\alpha \zeta}^{\sigma}\right] \mathbf{e}^{\sigma, \zeta^{\dagger}} \\
& -\mathbf{K}_{0}^{-1} p_{j} \mathbf{R}_{j}\left[\nabla_{\mathbf{p}} \mathbf{e}^{\sigma, \alpha}\right] \cdot\left[\nabla_{\mathbf{x}} \bar{W}_{\alpha \zeta}^{\sigma}\right] \mathbf{e}^{\sigma, \zeta^{\dagger}} \\
= & \nabla_{\mathbf{p}} \Omega^{\sigma} \cdot \nabla_{\mathbf{x}} \bar{W}_{\alpha \zeta}^{\sigma} \mathbf{e}^{\sigma, \alpha} \mathbf{e}^{\sigma, \zeta^{\dagger}}+\left(\Omega^{\sigma}-\mathbf{K}_{0}^{-1} p_{j} \mathbf{R}_{j}\right) \\
& \times\left[\nabla_{\mathbf{p}} \mathbf{e}^{\sigma, \alpha}\right] \cdot\left[\nabla_{\mathbf{x}} \bar{W}_{\alpha \zeta}^{\sigma}\right] \mathbf{e}^{\sigma, \zeta \dagger} .
\end{aligned}
$$

Upon the operation $\operatorname{Tr}\left[\mathbf{D}^{\tau, \xi \nu}(\cdot)\right]$ the second term vanishes, while the first term reduces to $\nabla_{\mathbf{p}} \Omega^{\tau} \cdot \nabla_{\mathbf{x}} \bar{W}_{\xi \nu}^{\tau}$ by Eq. (28) and the fact that $\mathbf{d}^{\tau, \xi}$ is a left eigenvector of the matrix $\mathbf{K}_{0}^{-1} p_{j} \mathbf{R}_{j}$ with the eigenvalue $\Omega^{\tau}$.

The other term, $\operatorname{Tr}\left[\mathbf{D}^{\tau, \xi \nu^{\dagger}} \partial_{x_{j}} \overline{\mathbf{W}} \mathbf{R}_{j} \mathbf{K}_{0}^{-1}\right]$, gives the identical result.

\section{Scattering Kernel}

The $(s, j)$ element of the matrix

$$
\begin{aligned}
\langle\mathbf{F}\rangle+ & \mathbf{K}_{0}^{-1} \mathbf{R}_{j} \frac{\partial}{\partial x_{j}} \overline{\mathbf{W}}+\frac{\partial}{\partial x_{j}} \overline{\mathbf{W}} \mathbf{R}_{j} \mathbf{K}_{0}^{-1} \\
= & 2 i \int d \mathbf{q}\left\langle e^{i \mathbf{q} \mathbf{\mathbf { x }} / \omega_{1}} \hat{\mathbf{V}}(\mathbf{q}) \mathbf{W}_{1}\left(\mathbf{p}-\frac{\mathbf{q}}{2 \omega_{1}}\right)\right. \\
& \left.-\mathbf{W}_{1}\left(\mathbf{p}-\frac{\mathbf{q}}{2 \omega_{2}}\right) \hat{\mathbf{V}}^{\dagger}(\mathbf{q}) e^{-i \mathbf{q}^{\dagger} \tilde{\mathbf{x}} / \omega_{2}}\right\rangle
\end{aligned}
$$

has the expression

$$
\begin{aligned}
& \sum_{\sigma, \alpha, \zeta, \eta} 2 i \omega_{1}^{3} \int \mathrm{d} \mathbf{k}\left(\Omega^{\sigma}(\mathbf{p}+\mathbf{k})-\Omega^{\sigma}(\mathbf{p})-i \ell\right)^{-1} d_{f}^{\sigma, \alpha^{*}}(\mathbf{p}+\mathbf{k}) \Psi_{f g s i}\left(\omega_{1} \mathbf{k}\right) \bar{W}_{\eta \zeta}^{\sigma}(\mathbf{p}) e_{g}^{\sigma, \eta}(\mathbf{p}) E_{i j}^{\sigma, \alpha \zeta}(\mathbf{p}+\mathbf{k}, \mathbf{p})-2 i \omega_{1}^{3} \\
& \quad \times \int \mathrm{d} \mathbf{k}\left(\Omega^{\sigma}\left(\mathbf{p}+\frac{1}{2}\left(\frac{\omega_{2}}{\omega_{1}}+1\right) \mathbf{k}\right)-\Omega^{\sigma}\left(\mathbf{p}+\frac{1}{2}\left(\frac{\omega_{2}}{\omega_{1}}-1\right) \mathbf{k}\right)-i \ell\right)^{-1} \\
& \times e^{i\left(1-\omega_{2} / \omega_{1}\right) \mathbf{k}^{\top} \tilde{\mathbf{x}}} \bar{W}_{\alpha \eta}^{\sigma}\left(\mathbf{p}+\frac{1}{2}\left(1+\frac{\omega_{2}}{\omega_{1}}\right) \mathbf{k}\right) \Phi_{f g s i}^{*}\left(-\omega_{2} \mathbf{k}\right) \\
& \quad \times e_{g}^{\sigma, \eta^{*}}\left(\mathbf{p}+\frac{1}{2}\left(1+\frac{\omega_{2}}{\omega_{1}}\right) \mathbf{k}\right) d_{f}^{\sigma, \zeta}\left(\mathbf{p}+\frac{1}{2}\left(\frac{\omega_{2}}{\omega_{1}}-1\right) \mathbf{k}\right) \\
& \quad \times E_{i j}^{\sigma, \alpha \zeta}\left(\mathbf{p}+\frac{1}{2}\left(1+\frac{\omega_{2}}{\omega_{1}}\right) \mathbf{k}, \mathbf{p}+\frac{1}{2}\left(\frac{\omega_{2}}{\omega_{1}}-1\right) \mathbf{k}\right)-2 i \omega_{2}^{3} \int \mathrm{d} \mathbf{k}\left(\Omega^{\sigma}\left(\mathbf{p}+\frac{1}{2}\left(1-\frac{\omega_{1}}{\omega_{2}}\right) \mathbf{k}\right)\right.
\end{aligned}
$$




$$
\begin{aligned}
& \left.-\Omega^{\sigma}\left(\mathbf{p}-\frac{1}{2}\left(\frac{\omega_{1}}{\omega_{2}}+1\right) \mathbf{k}\right)-i \ell\right)^{-1} e^{i\left(1-\omega_{1} / \omega_{2}\right) \mathbf{k}^{\dagger} \tilde{\mathbf{x}}} \bar{W}_{\eta, \zeta}^{\sigma}\left(\mathbf{p}-\frac{1}{2}\left(\frac{\omega_{1}}{\omega_{2}}+1\right) \mathbf{k}\right) \Phi_{f g j n}\left(\omega_{1} \mathbf{k}\right) \\
& \times d_{f}^{\sigma, \alpha^{*}}\left(\mathbf{p}+\frac{1}{2}\left(1-\frac{\omega_{1}}{\omega_{2}}\right) \mathbf{k}\right) e_{g}^{\sigma, \eta}\left(\mathbf{p}-\frac{1}{2}\left(\frac{\omega_{1}}{\omega_{2}}+1\right) \mathbf{k}\right) E_{s n}^{\sigma, \alpha \zeta}\left(\mathbf{p}+\frac{1}{2}\left(1-\frac{\omega_{1}}{\omega_{2}}\right) \mathbf{k}, \mathbf{p}-\frac{1}{2}\left(\frac{\omega_{1}}{\omega_{2}}+1\right) \mathbf{k}\right) \\
& +2 i \omega_{2}^{3} \int \mathrm{d} \mathbf{k}\left(\Omega^{\sigma}(\mathbf{p})-\Omega^{\sigma}(\mathbf{p}-\mathbf{k})-i \ell\right)^{-1} \bar{W}_{\alpha \eta}^{\sigma}(\mathbf{p}) \Psi_{f g j n}^{*}\left(-\omega_{2} \mathbf{k}\right) e_{g}^{\sigma, \eta^{*}}(\mathbf{p}) d_{f}^{\sigma, \zeta}(\mathbf{p}-\mathbf{k}) E_{s n}^{\sigma, \alpha \zeta}(\mathbf{p}, \mathbf{p}-\mathbf{k}) .
\end{aligned}
$$

Using the identity

$$
\lim _{\ell \rightarrow 0} \frac{1}{x-i \ell}=i \pi \delta(x)+\frac{1}{x}
$$

and the symmetry properties (15), (16), and (27), we obtain in the limit $\ell \rightarrow 0$ Eq. (46) from Eq. (41).

\section{ACKNOWLEDGMENTS}

This research is supported by Defense Advanced Research Projects Agency (DARPA) grant N00014-02-1-0603.

\section{REFERENCES}

1. A. C. Fannjiang, "Two-frequency radiative transfer and asymptotic solution," J. Opt. Soc. Am. A 24, 2248-2256 (2007).

2. M. Mishchenko, L. Travis, and A. Lacis, Multiple Scattering of Light by Particles: Radiative Transfer and Coherent Backscattering (Cambridge U. Press, 2006).

3. L. Mandel and E. Wolf, Optical Coherence and Quantum Optics (Cambridge U. Press, 1995).

4. A. C. Fannjiang, "Information transfer in disordered media by broadband time reversal: stability, resolution and capacity," Nonlinearity 19, 2425-2439 (2006).

5. A. C. Fannjiang and P. M. Yan, "Multi-frequency imaging of multiple targets in Rician fading channels: stability and resolution," Inverse Probl. 23, 1801-1819 (2007).

6. A. C. Fannjiang, "Space-frequency correlation of classical waves in disordered media: high-frequency and small scale asymptotics," 80, 14005 (2007).

7. I. V. Lindell, A. H. Sihvola, S. A. Tretyakov, and A. J. Viitanen, Electromagnetic Waves in Chiral and Bi-isotropic Media (Artech House, 1994).
8. T. H. O'Dell, The Electrodynamics of Magneto-Electric Media (North-Holland, 1970).

9. L. D. Landau, E. M. Lifshitz, and L. P. Pitaevskii, Electrodynamics of Continuous Media (Elsevier Butterworth-Heinemann, 1984).

10. M. Born and W. Wolf, Principles of Optics, 7th (expanded) ed. (Cambridge U. Press, 1999).

11. W. S. Weiglhofer and A. Lakhtakia, Introduction to Complex Mediums for Optics and Electromagnetics (SPIE Press, 2003).

12. D. K. Cheng and 1. A. Kong, "Covariant descriptions of bianisotropic media," Proc. IEEE 56, 248-251 (1968).

13. A. C. Fannjiang, "Mutual coherence of polarized light in disordered media: two-frequency method extended," submitted to J. Phys. A: Math. Theor. 40, 13667-13683 (2007).

14. H. N. Kritikos, N. Engheta, and D. L. Jaggard, "Symmetry in electromagnetic media: a spinor viewpoint," in Electromagnetic Symmetry, C. E. Baum and H. N. Kritikos, eds. (Taylor \& Francis, 1995), pp. 185-230.

15. L. Ryzhik, G. Papanicolaou, and J. B. Keller, "Transport equations for elastic and other waves in random media," Wave Motion 24, 327-370 (1996).

16. I. Bialynicki-Birula, "Photon wave function," Prog. Opt. 36, 245-294 (1996).

17. V. G. Veselago, "The electrodynamics of substances with simultaneously negative values of $\epsilon$ and $\mu$," Sov. Phys. Usp. 10, 509-514 (1968).

18. D. R. Smith, W. Padilla, D. C. Vier, S. C. Nemat-Nasser, and S. Schultz, "A composite medium with simultaneously negative permeability and permittivity," Phys. Rev. Lett. 84, 4184-4187 (2000)

19. D. R. Smith, J. B. Pendry, and M. C. K. Wiltshire, "Metamaterials and negative refractive index," Science 305, 788-792 (2004).

20. S. Chandrasekhar, Radiative Transfer (Dover, 1960).

21. A. Kokhanovsky, Polarization Optics of Random Media (Springer, 2003). 